PHYSICAL REVIEW D 80, 036003 (2009)

\title{
Complete reduction of one-loop tensor 5- and 6-point integrals
}

\author{
Th. Diakonidis T. Riemann and J. B. Tausk \\ Deutsches Elektronen-Synchrotron, DESY, Platanenallee 6, 15738 Zeuthen, Germany \\ J. Fleischer \\ Fakultät für Physik, Universität Bielefeld, Universitätsstrasse 25, 33615 Bielefeld, Germany \\ J. Gluza and K. Kajda \\ Institute of Physics, University of Silesia, Uniwersytecka 4, 40-007 Katowice, Poland
}

(Received 24 February 2009; published 5 August 2009)

\begin{abstract}
We perform a complete analytical reduction of general one-loop Feynman integrals with five and six external legs for tensors up to rank $R=3$ and 4, respectively. An elegant formalism with extensive use of signed minors is developed for the cancellation of inverse Gram determinants. The 6-point tensor functions of rank $R$ are expressed in terms of 5-point tensor functions of rank $R-1$, and the latter are reduced to scalar four-, three-, and two-point functions. The resulting compact formulas allow both for a study of analytical properties and for efficient numerical programming. They are implemented in FORTRAN and MATHEMATICA.
\end{abstract}

DOI: 10.1103/PhysRevD.80.036003

PACS numbers: $11.80 . \mathrm{Cr}, 12.38 . \mathrm{Bx}$

\section{INTRODUCTION}

At the proton-proton collider LHC and the planned $e^{+} e^{-}$collider ILC, a large number of particles per event may be produced. The hope is to discover one or several Higgs bosons or supersymmetric particles, which are typically expected to be quite heavy. The interest is also directed to the study of known massive particles like the $W$ and $Z$ bosons or the top quark. Since the production rates are large, a proper description of the cross sections will typically include one-loop corrections to $n$-particle reactions, where some of the final state particles may be massive.

The Feynman integrals for reactions with up to four external particles have been systematically studied and evaluated in numerous studies. We just want to mention here the seminal papers $[1,2]$ and theFORTRAN packages FF [3] and LOOPTOOLS [4], which represent the state of the art until now. The treatment of Feynman integrals with a higher multiplicity than four becomes quite involved if questions of efficiency and stability become vital, as it happens with the calculational problems related to highdimensional phase-space integrals over sums of thousands of Feynman diagrams with internal loops.

In this article, we will concentrate on the evaluation of massive one-loop Feynman integrals with $n$ external legs and some tensor structure,

$$
I_{n}^{\mu_{1} \cdots \mu_{R}}=\int \frac{d^{d} k}{i \pi^{d / 2}} \frac{\prod_{r=1}^{R} k^{\mu_{r}}}{\prod_{j=1}^{n} c_{j}^{\nu_{j}}},
$$

where the denominators $c_{j}$ have indices $\nu_{j}$ and chords $q_{j}$,

$$
c_{j}=\left(k-q_{j}\right)^{2}-m_{j}^{2}+i \varepsilon .
$$

We will study in the following the cases $n=5$ with $R \leq 3$ and $n=6$ with $R \leq 4$, and we will conventionally assume $q_{n}=0$. The space-time dimension is $d=4-2 \epsilon$.

There are several strategies one might follow. One is the reduction of higher-point tensor integrals to tensor integrals with less external lines and/or lower tensor rank [5$8]$; a second approach is essentially numerical $[9,10]$ or seminumerical [11-13]. A third one rests on the unitarity cut method [14-17]. In this case, a one-loop amplitude is evaluated as a whole, by using Cutkosky rules, instead of computing loop integrals from each of the Feynman diagrams. Very recently, the first NLO QCD corrections, in leading color approximation, to $W+3$-jet production at hadron colliders have been obtained $[18,19]$. We would like to refer to e.g. [20-24] for recent overviews on the subject.

Here, we will advocate yet another approach and reduce the tensor integrals algebraically to sums over a small set of scalar two-, three-, and four-point functions, which we assume to be known. Whether such a complete reduction is competitive with the other approaches might be disputed. Evidently, this depends on the specific problem under investigation. For a study of gauge invariance and of the ultraviolet (UV) and infrared (IR) singularity structure of a set of Feynman diagrams, it is evident that a complete reduction is advantageous, and it may also be quite useful for a tuned, analytical study of certain regions of potential numerical instabilities.

We have chosen a strictly algebraic approach and will rely heavily on the algebra of signed minors which was worked out in detail by Melrose in [25]. One of the basic observations of Melrose was that in four dimensions all the scalar integrals can be reduced to scalar 4-point functions 
and simpler ones. In [26], a representation of arbitrary oneloop tensor integrals in terms of scalar integrals was derived. The representation includes, however, scalar integrals with higher indices $\nu_{j}$ and higher space-time dimensions $d+2 l$. The subsequent reduction to scalar integrals with only the original indices and the generic space-time dimension $d$ is possible with the use of integration-by-parts identities [27] and generalizations of them with dimensional shifts. The latter have been derived in [28], and a systematical application to one-loop integrals may be found in $[29,30]$. A closely related scheme, applied to cases with massless internal lines, was presented in [31]. Basically, the reduction problem has been solved this way for $n$-point functions. There was one attempt to use the Davydychev-Tarasov reduction for the description of oneloop contributions to the process $e^{+} e^{-} \rightarrow H \nu \bar{\nu}$ [32], and the numerical problems due to the five-point functions were discussed in some detail. To a large extent they root in the appearance of inverse powers of Gram determinants. This feature of the Davydychev-Tarasov reduction was identified as disadvantageous soon after its derivation, e.g. in [33], where a strategy for avoiding these problems was developed. Besides the problem of inverse powers of the Gram determinant of the corresponding Feynman diagram, there are additional kinematical singularities related to subdiagrams. This will not be discussed here; we refer to e.g. $[5-8,13,17,33,34]$ and references therein.

In this article, we investigate the reduction of tensor integrals with five and six external legs which are of immediate importance in applications at the LHC. In Sec. II we represent tensor integrals by scalar integrals in shifted space-time dimensions with shifted indices. Sections III and IV contain our main result. In Sec. III we go one step further in the reduction of five-point tensors compared to [29] and demonstrate how to cancel all inverse powers of the Gram determinant appearing in the Davydychev-Tarasov reduction. Earlier results for tensors of rank two may be found in [35]. Section IV contains the reduction of tensorial six-point functions to tensorial 5point functions. The corresponding Gram determinant is identically zero $[6,8,29]$, and the reduction becomes quite compact. Some numerical results and a short discussion are given in Sec. V. The numerics is obtained with two independent implementations, one made in MATHEMATICA, and another one in FORTRAN. The MATHEMATICA program HEXAGON.M with the reduction formulas is made publicly available [36], see also [37] for a short description. For numerical applications, one has to link the package with a program for the evaluation of scalar one- to four-point functions, e.g. with LOOPTOOLS [3,4,38], CUTTOOLS $[12,39]$, QCDLOOP [40]. Appendices are devoted to some known, but necessary, details on Gram determinants and the algebra of signed minors and to a short summary about the reduction of dimensionally shifted four- and five-point integrals.

\section{REPRESENTING TENSOR INTEGRALS BY SCALAR INTEGRALS IN SHIFTED SPACE-TIME DIMENSIONS}

At first we give the reduction of tensor integrals to a set of scalar integrals for arbitrary n-point functions. Following [26,29], assuming here the indices of propagators to be equal to one, $\nu_{r}=1$, one has

$$
\begin{gathered}
I_{n}^{\mu}=\int^{d} k^{\mu} \prod_{r=1}^{n} c_{r}^{-1}=-\sum_{i=1}^{n-1} q_{i}^{\mu} I_{n, i}^{[d+]}, \\
I_{n}^{\mu \nu}=\int^{d} k^{\mu} k^{\nu} \prod_{r=1}^{n} c_{r}^{-1} \\
=\sum_{i, j=1}^{n-1} q_{i}^{\mu} q_{j}^{\nu} n_{i j} I_{n, i j}^{[d+]^{2}}-\frac{1}{2} g^{\mu \nu} I_{n}^{[d+]}, \\
I_{n}^{\mu \nu \lambda}=\int^{d} k^{\mu} k^{\nu} k^{\lambda} \prod_{r=1}^{n} c_{r}^{-1} \\
=-\sum_{i, j, k=1}^{n-1} q_{i}^{\mu} q_{j}^{\nu} q_{k}^{\lambda} n_{i j k} I_{n, i j k}^{[d+]^{3}} \\
+\frac{1}{2} \sum_{i=1}^{n-1}\left(g^{\mu \nu} q_{i}^{\lambda}+g^{\mu \lambda} q_{i}^{\nu}+g^{\nu \lambda} q_{i}^{\mu}\right) I_{n, i}^{[d+]^{2}}, \\
I_{n}^{\mu \nu \lambda \rho}=\int^{d} k^{\mu} k^{\nu} k^{\lambda} k^{\rho} \prod_{r=1}^{n} c_{r}^{-1} \\
=\sum_{i, j, k, l=1}^{n-1} q_{i}^{\mu} q_{j}^{\nu} q_{k}^{\lambda} q_{l}^{\rho} n_{i j k l} I_{n, i j k l}^{[d+]^{4}} \\
+\frac{1}{2} \sum_{i, j=1}^{n-1}\left(g^{\mu \nu} q_{i}^{\lambda} q_{j}^{\rho}+g^{\mu \lambda} q_{i}^{\nu} q_{j}^{\rho}+g^{\nu \lambda} q_{i}^{\mu} q_{j}^{\rho}\right. \\
\left.+g^{\mu \rho} q_{i}^{\nu} q_{j}^{\lambda}+g^{\nu \rho} q_{i}^{\mu} q_{j}^{\lambda}+g^{\lambda \rho} q_{i}^{\mu} q_{j}^{\nu}\right) n_{i j} I_{n, i j}^{[d+]^{3}} \\
+\frac{1}{4}\left(g^{\mu \nu} g^{\lambda \rho}+g^{\mu \lambda} g^{\nu \rho}+g^{\mu \rho} g^{\nu \lambda}\right) I_{n}^{[d+]^{2}},
\end{gathered}
$$

where $[d+]$ is an operator shifting the space-time dimension by two units and

$$
\begin{aligned}
I_{p, i j k \cdots}^{[d+]^{l}, s t u} & =\int^{[d+]^{l}} \prod_{r=1}^{n} \frac{1}{c_{r}^{1+\delta_{r i}+\delta_{r j}+\delta_{r k}+\cdots-\delta_{r s}-\delta_{r t}-\delta_{r u}-\cdots},} \\
\int^{d} & \equiv \int \frac{d^{d} k}{i \pi^{d / 2}},
\end{aligned}
$$

where $[d+]^{l}=4+2 l-2 \epsilon$ (observe that $p$ is the number of scalar propagators of the " $p$-point function" and that equal lower and upper indices cancel, $p \leq n$ ). In (2.2), (2.3), and (2.4), the coefficients $n_{i j}, n_{i j k}$ and $n_{i j k l}$ were introduced. These stand for the product of factorials of the number of equal indices: e.g. $n_{i i i i}=4$ !, $n_{i j i i}=3$ !, $n_{i i j j}=2 ! 2 !, n_{i j k k}=2 !, n_{i j k l}=1$ ! (indices $i, j, k, l$ all 
different from each other). Of particular relevance are the following relations for the successive application of recurrence relations to reduce higher dimensional integrals:

$$
n_{i j}=\nu_{i j}, \quad n_{i j k}=\nu_{i j} \nu_{i j k}, \quad n_{i j k l}=\nu_{i j} \nu_{i j k} \nu_{i j k l},
$$

where

$$
\begin{gathered}
\nu_{i j}=1+\delta_{i j}, \quad \nu_{i j k}=1+\delta_{i k}+\delta_{j k}, \\
\nu_{i j k l}=1+\delta_{i l}+\delta_{j l}+\delta_{k l} .
\end{gathered}
$$

In the next step the integrals in higher dimension have to be reduced to integrals in generic dimension. Here particular attention has to be paid to $I_{5}^{[d+]}$. Reducing the tensor integrals, this term drops out in general [7,41].

\section{PENTAGONS}

We start with the reduction of the pentagons. This will also provide the basis for calculating the hexagons as we shall see.

\section{A. Scalar and vector integrals}

For the scalar 5-point function the recursion relation (I.31) [30] reads for $n=5$

$$
(d-4)()_{5} I_{5}^{[d+]}=\left(\begin{array}{l}
0 \\
0
\end{array}\right)_{5} I_{5}-\sum_{s=1}^{5}\left(\begin{array}{l}
0 \\
s
\end{array}\right)_{5} I_{4}^{s} .
$$

This is an exact identity between dimensionally regularized integrals, no approximation has been made so far. The integral $I_{5}^{[d+]}$ contains neither ultraviolet nor infrared divergences and is therefore finite in the limit $d \rightarrow 4$. Hence, the left-hand side of (3.1) is of order $\epsilon$. Neglecting this term,

$$
E \equiv I_{5}=\frac{1}{\left(\begin{array}{l}
0 \\
0
\end{array}\right)_{5}} \sum_{s=1}^{5}\left(\begin{array}{l}
0 \\
s
\end{array}\right)_{5} I_{4}^{s},
$$

i.e. the scalar five-point function is expressed in the limit $d \rightarrow 4$ in terms of scalar four-point functions, which are obtained by scratching in the five terms of the sum the sth scalar propagator, respectively. This was already derived in [25], see Eq. (6.1) there. Note that, depending on the kinematical configuration and on the masses of the internal lines, $I_{5}$ and $I_{4}^{S}$ can be infrared divergent in some cases.

Similarly, for the tensor integral of rank $R=1$ (vector) in (2.1) we obtain:

$$
I_{5}^{\mu}=\sum_{i=1}^{4} q_{i}^{\mu} I_{5, i}
$$

with

$$
\begin{aligned}
I_{5, i} & \equiv E_{i}=-I_{5, i}^{[d+]} \\
& =(d-4) \frac{\left(\begin{array}{l}
0 \\
i
\end{array}\right)_{5}}{\left(\begin{array}{l}
0 \\
0
\end{array}\right)_{5}} I_{5}^{[d+]}-\frac{1}{\left(\begin{array}{l}
0 \\
0
\end{array}\right)_{5}} \sum_{s=1}^{5}\left(\begin{array}{ll}
0 & i \\
0 & s
\end{array}\right)_{5} I_{4}^{s},
\end{aligned}
$$

where again in the limit $d \rightarrow 4$ the $I_{5}^{[d+]}$ disappears [42]. These two cases are simple and lead to a direct reduction to scalar integrals, without the Gram determinant ()$_{5}$ occurring anyway. In the following we want to reduce tensor integrals of higher rank and show, like in $[5,8]$, that also in these cases the Gram determinant can be cancelled.

\section{B. $R=\mathbf{2}$ tensor integrals}

The tensor integral of rank 2 can be written without a $g_{\mu \nu}$ term:

$$
I_{5}^{\mu \nu}=\sum_{i, j=1}^{4} q_{i}^{\mu} q_{j}^{\nu} I_{5, i j}
$$

which is obtained by replacing $g_{\mu \nu}$ by

$$
g^{\mu \nu}=2 \sum_{i, j=1}^{4} \frac{\left(\begin{array}{l}
i \\
j
\end{array}\right)_{5}}{()_{5}} q_{i}^{\mu} q_{j}^{\nu}
$$

(assuming $q_{1} \cdots q_{4}$ 4-dimensional and independent) and further by reducing the integrals in (2.2) to generic dimension. This applies in the same manner also for the tensor integrals of higher rank. Reducing the integrals of highest dimension, recursion relation (I.30) is used. For the 5-point function several cases have been worked out in: (I.41), (I.42), and (I.43). For completeness we give in the appendix explicitly the cases needed in the present work.

One remark is needed concerning the integral $I_{5}^{[d+]}$. It is known $[7,41]$ that it always cancels in the end. This provides a very useful check on our calculations, which we have performed in every particular case under consideration. Anticipating this cancellation, we will, for the ease of our discussion, drop terms proportional to $I_{5}^{[d+]}$ wherever they appear in the following derivation. With this in mind we can write for $I_{5, i j}$ in (2.2) with (B2):

$$
\begin{aligned}
I_{5, i j} & =\nu_{i j} I_{5, i j}^{[d+]^{2}} \\
& =-\frac{\left(\begin{array}{l}
0 \\
j
\end{array}\right)_{5}}{()_{5}} I_{5, i}^{[d+]}+\sum_{s=1, s \neq i}^{5} \frac{\left(\begin{array}{c}
s \\
j
\end{array}\right)_{5}}{()_{5}} I_{4, i}^{[d+], s} \\
& =\frac{\left(\begin{array}{l}
0 \\
j
\end{array}\right)_{5}}{()_{5}} I_{5, i}+\sum_{s=1, s \neq i}^{5} \frac{\left(\begin{array}{l}
s \\
j
\end{array}\right)_{5}}{()_{5}} I_{4, i}^{[d+], s},
\end{aligned}
$$

and by means of (B3) we obtain: 


$$
\begin{aligned}
I_{5, i j}= & \frac{1}{\left.\left(\begin{array}{l}
0 \\
0
\end{array}\right)_{5}\right)_{5}} \sum_{s=1, s \neq i}^{5} \frac{1}{\left(\begin{array}{l}
s \\
s
\end{array}\right)_{5}}\left\{-\left(\begin{array}{l}
0 \\
j
\end{array}\right)_{5}\left(\begin{array}{ll}
0 & s \\
0 & i
\end{array}\right)_{5}\left(\begin{array}{l}
s \\
s
\end{array}\right)_{5}-\left(\begin{array}{l}
s \\
j
\end{array}\right)_{5}\left(\begin{array}{ll}
0 & s \\
i & s
\end{array}\right)_{5}\left(\begin{array}{l}
0 \\
0
\end{array}\right)_{5}+\left(\begin{array}{l}
s \\
0
\end{array}\right)_{5}\left(\begin{array}{ll}
0 & s \\
0 & s
\end{array}\right)_{5}\left(\begin{array}{l}
i \\
j
\end{array}\right)_{5}\right\} I_{4}^{s} \\
& -\frac{\left(\begin{array}{l}
i \\
j
\end{array}\right)_{5}}{\left.\left(\begin{array}{l}
0 \\
0
\end{array}\right)_{5}\right)_{5}} \sum_{s=1, s \neq i}^{5} \frac{1}{\left(\begin{array}{l}
s \\
s
\end{array}\right)_{5}}\left(\begin{array}{l}
s \\
0
\end{array}\right)_{5}\left(\begin{array}{ll}
0 & s \\
0 & s
\end{array}\right)_{5} I_{4}^{s}-\frac{1}{\left(\begin{array}{l}
0 \\
0
\end{array}\right)_{5}()_{5}} \sum_{s, t=1, s \neq i, t}^{5} \frac{1}{\left(\begin{array}{l}
s \\
s
\end{array}\right)_{5}}\left\{-\left(\begin{array}{l}
0 \\
j
\end{array}\right)_{5}\left(\begin{array}{ll}
t & s \\
0 & i
\end{array}\right)_{5}\left(\begin{array}{l}
s \\
s
\end{array}\right)_{5}\right. \\
& \left.-\left(\begin{array}{l}
s \\
j
\end{array}\right)_{5}\left(\begin{array}{ll}
t & s \\
i & s
\end{array}\right)_{5}\left(\begin{array}{l}
0 \\
0
\end{array}\right)_{5}+\left(\begin{array}{l}
s \\
0
\end{array}\right)_{5}\left(\begin{array}{ll}
t & s \\
0 & s
\end{array}\right)_{5}\left(\begin{array}{l}
i \\
j
\end{array}\right)_{5}\right\}_{3}^{s t}+\frac{\left(\begin{array}{l}
i \\
j
\end{array}\right)_{5}}{\left.\left(\begin{array}{l}
0 \\
0
\end{array}\right)_{5}\right)_{5}} \sum_{s, t=1, s \neq i, t}^{5} \frac{1}{s}\left(\begin{array}{l}
s \\
s
\end{array}\right)_{5}
\end{aligned}
$$

Using (3.6) again, we find

$$
\begin{aligned}
& I_{5}^{\mu \nu}=\sum_{i, j=1}^{4} q_{i}^{\mu} q_{j}^{\nu} E_{i j}+g^{\mu \nu} E_{00}, \\
& E_{i j}=\sum_{s=1}^{5} S_{i j}^{4, s} I_{4}^{s}+\sum_{s, t=1}^{5} S_{i j}^{3, s t} I_{3}^{s t},
\end{aligned}
$$

where

$$
\begin{gathered}
S_{i j}^{4, s}=\frac{1}{\left(\begin{array}{l}
0 \\
0
\end{array}\right)_{5}} \sum_{s=1}^{5} \frac{1}{\left(\begin{array}{l}
s \\
s
\end{array}\right)_{5}} X_{i j}^{s 0}, \\
S_{i j}^{3, s t}=-\frac{1}{\left(\begin{array}{l}
0 \\
0
\end{array}\right)_{5}} \sum_{s, t=1}^{5} \frac{1}{\left(\begin{array}{l}
s \\
s
\end{array}\right)_{5}} X_{i j}^{s t}
\end{gathered}
$$

and $X_{i j}^{s 0}$ and $X_{i j}^{s t}$ are defined in (A22). Finally,

$$
\begin{aligned}
E_{00}= & -\frac{1}{2} \frac{1}{\left(\begin{array}{l}
0 \\
0
\end{array}\right)_{5}} \sum_{s=1}^{5} \frac{\left(\begin{array}{l}
s \\
0
\end{array}\right)_{5}}{\left(\begin{array}{ll}
s \\
s
\end{array}\right)_{5}}\left[\left(\begin{array}{ll}
0 & s \\
0 & s
\end{array}\right)_{5} I_{4}^{s}\right. \\
& \left.-\sum_{t=1}^{5}\left(\begin{array}{ll}
t & s \\
0 & s
\end{array}\right)_{5} I_{3}^{s t}\right] .
\end{aligned}
$$

In this way we have cancelled the Gram determinant for the tensor of rank 2. For later reference, we note that, by taking into account (B4), we can also write

$$
E_{00}=-\frac{1}{2} \frac{1}{\left(\begin{array}{l}
0 \\
0
\end{array}\right)_{5}} \sum_{s=1}^{5}\left(\begin{array}{l}
s \\
0
\end{array}\right)_{5} I_{4}^{[d+], s} .
$$

\section{C. $\boldsymbol{R}=\mathbf{3}$ tensor integrals}

The tensor integral of rank 3 can be written as:

$$
I_{5}^{\mu \nu \lambda}=\sum_{i, j, k=1}^{4} q_{i}^{\mu} q_{j}^{\nu} q_{k}^{\lambda} I_{5, i j k} .
$$

We will now rewrite this into another representation, thereby avoiding Gram determinants ()$_{5}$ in the denominators of the new tensor coefficients $E_{i j k}, E_{00 k}$ :

$$
\begin{gathered}
I_{5}^{\mu \nu \lambda}=\sum_{i, j, k=1}^{4} q_{i}^{\mu} q_{j}^{\nu} q_{k}^{\lambda} E_{i j k}+\sum_{k=1}^{4} g^{[\mu \nu} q_{k}^{\lambda]} E_{00 k}, \\
E_{i j k}=\sum_{s=1}^{5} S_{i j k}^{4, s} I_{4}^{s}+\sum_{s, t=1}^{5} S_{i j k}^{3, s t} I_{3}^{S t}+\sum_{s, t, u=1}^{5} S_{i j k}^{2, s t u} I_{2}^{s t u} .
\end{gathered}
$$

According to (2.3) we have with (3.6):

$$
\begin{aligned}
I_{5, i j k}= & -\nu_{i j} \nu_{i j k} I_{5, i j k}^{[d+]^{3}}+\frac{\left(\begin{array}{c}
j \\
k
\end{array}\right)_{5}}{()_{5}} I_{5, i}^{[d+]^{2}}+\frac{\left(\begin{array}{c}
i \\
k
\end{array}\right)_{5}}{()_{5}} I_{5, j}^{[d+]^{2}} \\
& +\frac{\left(\begin{array}{c}
i \\
j
\end{array}\right)_{5}}{()_{5}} I_{5, k}^{[d+]^{2}} .
\end{aligned}
$$

By means of recursion (B1), taking into account (3.7) and keeping in mind to drop $I_{5}^{[d+]}$, we have: 


$$
\begin{aligned}
I_{5, i j k}= & \frac{\left(\begin{array}{l}
0 \\
k
\end{array}\right)_{5}}{()_{5}} \nu_{i j} I_{5, i j}^{[d+]^{2}}-\sum_{s=1, s \neq i, j}^{5} \frac{\left(\begin{array}{l}
s \\
k
\end{array}\right)_{5}}{()_{5}} \nu_{i j} I_{4, i j}^{[d+]^{2}, s} \\
& +\frac{\left(\begin{array}{l}
i \\
j
\end{array}\right)_{5}}{()_{5}} \sum_{s=1}^{5} \frac{\left(\begin{array}{l}
s \\
k
\end{array}\right)_{5}}{()_{5}} I_{4}^{[d+], s} \\
= & \frac{\left(\begin{array}{l}
0 \\
k
\end{array}\right)_{5}}{()_{5}} I_{5, i j}+\frac{\left(\begin{array}{l}
i \\
j
\end{array}\right)_{5}}{()_{5}} \sum_{s=1}^{5} \frac{\left(\begin{array}{l}
s \\
k
\end{array}\right)_{5}}{()_{5}} I_{4}^{[d+], s} \\
& -\sum_{s=1, s \neq i, j}^{5} \frac{\left(\begin{array}{l}
s \\
k
\end{array}\right)_{5}}{()_{5}} \nu_{i j} I_{4, i j}^{[d+]^{2}, s} .
\end{aligned}
$$

Collecting the terms proportional to ()$_{5}^{-2}$ we have with

$$
I_{5, i j}=\cdots+2 \frac{\left(\begin{array}{l}
i \\
j
\end{array}\right)_{5}}{()_{5}} E_{00}
$$

and (3.14):

$$
\begin{aligned}
& \frac{\left(\begin{array}{l}
i \\
j
\end{array}\right)_{5}}{\left(\begin{array}{l}
0 \\
0
\end{array}\right)_{5}} \frac{1}{()_{5}^{2}} \sum_{s=1}^{5}\left[\left(\begin{array}{l}
0 \\
0
\end{array}\right)_{5}\left(\begin{array}{l}
s \\
k
\end{array}\right)_{5}-\left(\begin{array}{l}
0 \\
k
\end{array}\right)_{5}\left(\begin{array}{l}
s \\
0
\end{array}\right)_{5}\right]_{4}^{[d+], s} \\
& =\frac{\left(\begin{array}{l}
i \\
j
\end{array}\right)_{5}}{\left(\begin{array}{l}
0 \\
0
\end{array}\right)_{5}} \frac{1}{()_{5}} \sum_{s=1}^{5}\left(\begin{array}{ll}
0 & s \\
0 & k
\end{array}\right)_{5} I_{4}^{[d+], s},
\end{aligned}
$$

i.e. we have already canceled one Gram determinant. We multiply (3.19) by

$$
\left(\begin{array}{l}
0 \\
0
\end{array}\right)_{5}
$$

such that we can make use of

$$
\left.\left(\begin{array}{l}
0 \\
0
\end{array}\right)_{5}\left(\begin{array}{l}
s \\
k
\end{array}\right)_{5}=\left(\begin{array}{ll}
0 & s \\
0 & k
\end{array}\right)_{5}\right)_{5}+\left(\begin{array}{l}
s \\
0
\end{array}\right)_{5}\left(\begin{array}{l}
0 \\
k
\end{array}\right)_{5}
$$

which will give us another factor ()$_{5}$. Adding all contributions, we obtain

$$
\begin{aligned}
& 3\left(\begin{array}{l}
0 \\
0
\end{array}\right)_{5} I_{5, i j k}=\sum^{\prime} \frac{1}{()_{5}\left(\begin{array}{l}
s \\
s
\end{array}\right)_{5}^{2}}\left\{\left(\begin{array}{l}
0 \\
k
\end{array}\right)_{5}\left(\begin{array}{l}
s \\
s
\end{array}\right)_{5}\left[X_{i j}^{s} I_{4}^{s}-X_{i j}^{s t} I_{3}^{s t}\right]+\left(\begin{array}{l}
i \\
j
\end{array}\right)_{5}\left(\begin{array}{l}
s \\
s
\end{array}\right)_{5}\left(\begin{array}{ll}
0 & s \\
0 & k
\end{array}\right)_{5}\left[\left(\begin{array}{ll}
0 & s \\
0 & s
\end{array}\right)_{5} I_{4}^{s}-\left(\begin{array}{ll}
t & s \\
0 & s
\end{array}\right)_{5} I_{3}^{s t}\right]\right. \\
& -\left(\begin{array}{l}
0 \\
0
\end{array}\right)_{5}\left(\begin{array}{l}
s \\
k
\end{array}\right)_{5}\left(\left[\left(\begin{array}{ll}
0 & s \\
i & s
\end{array}\right)_{5}\left(\begin{array}{ll}
0 & s \\
j & s
\end{array}\right)_{5}+\left(\begin{array}{ll}
i & s \\
j & s
\end{array}\right)_{5}\left(\begin{array}{ll}
0 & s \\
0 & s
\end{array}\right)_{5}\right] I_{4}^{s}\right.
\end{aligned}
$$

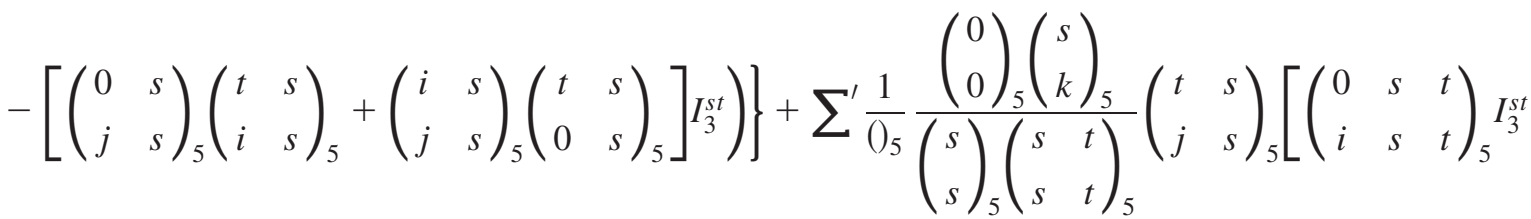

$$
\begin{aligned}
& \left.-\left(\begin{array}{ccc}
u & s & t \\
i & s & t
\end{array}\right)_{5} I_{2}^{s t u}\right]+(i \leftrightarrow k)+(j \leftrightarrow k) \\
& \equiv A+\sum^{\prime}\left\{\frac{1}{()_{5}\left(\begin{array}{l}
s \\
s
\end{array}\right)_{5}^{2}}\left(\begin{array}{l}
i \\
j
\end{array}\right)_{5}\left(\begin{array}{l}
s \\
s
\end{array}\right)_{5}\left(\begin{array}{ll}
0 & s \\
0 & k
\end{array}\right)_{5}\left[\left(\begin{array}{ll}
0 & s \\
0 & s
\end{array}\right)_{5} I_{4}^{s}-\left(\begin{array}{ll}
t & s \\
0 & s
\end{array}\right)_{5} I_{3}^{s t}\right]+\frac{1}{()_{5}} \frac{\left(\begin{array}{l}
0 \\
0
\end{array}\right)_{5}\left(\begin{array}{l}
s \\
k
\end{array}\right)_{5}}{\left(\begin{array}{ll}
s \\
s
\end{array}\right)_{5}\left(\begin{array}{ll}
s & t \\
s & t
\end{array}\right)_{5}}\left(\begin{array}{ll}
t \\
j & s
\end{array}\right)_{5}\right. \\
& \left.\times\left[\left(\begin{array}{ccc}
0 & s & t \\
i & s & t
\end{array}\right)_{5} I_{3}^{s t}-\left(\begin{array}{ccc}
u & s & t \\
i & s & t
\end{array}\right)_{5} I_{2}^{s t u}\right]\right\}+(i \leftrightarrow k)+(j \leftrightarrow k)
\end{aligned}
$$

The symbol $\Sigma^{\prime}$ in these equations denotes a sum $\sum_{s, t, u=1}^{5}$ in terms proportional to $I_{2}^{s t u}, \sum_{s, t=1}^{5}$ in terms proportional to $I_{3}^{s t}$, and $\sum_{s=1}^{5}$ in terms proportional to $I_{4}^{s}$. Concerning the symmetrization in (3.22), we point out that the original expression (3.18) is obviously symmetric under $(i \leftrightarrow j)$, while this is not explicitly seen in (3.22) anymore.
Later on, however, this symmetry will become apparent again.

All terms with factors of the type

$$
\left(\begin{array}{l}
i \\
j
\end{array}\right)_{5}
$$


can be considered, due to (3.6), as belonging to some $g_{\mu \nu}$ term. For other terms we have to use (3.21), which yields terms with ()$_{5}$ to be canceled. These are explicitly given in the coefficients of $I_{4}^{s}, I_{3}^{s t}$, and $I_{2}^{s t u}$, i.e. (3.30), (3.32), and (3.33). Inserting $X_{i j}^{s}$ and $X_{i j}^{s t}$, the remaining contributions to the coefficients of $I_{4}^{s}$ and $I_{3}^{s t}$ in (3.22) can be written as

$$
\begin{aligned}
& A=-\sum_{s=1}^{5} \frac{1}{()_{5}\left(\begin{array}{l}
s \\
s
\end{array}\right)_{5}^{2}}\left(\begin{array}{l}
0 \\
k
\end{array}\right)_{5}\left\{\left(\begin{array}{l}
s \\
s
\end{array}\right)_{5}\left[\left(\begin{array}{ll}
0 & s \\
0 & i
\end{array}\right)_{5}\left(\begin{array}{ll}
0 & s \\
j & s
\end{array}\right)_{5}-\left(\begin{array}{ll}
0 & j \\
s & i
\end{array}\right)_{5}\left(\begin{array}{ll}
0 & s \\
0 & s
\end{array}\right)_{5}\right]+\left(\begin{array}{l}
s \\
0
\end{array}\right)_{5}\left[\left(\begin{array}{ll}
0 & s \\
i & s
\end{array}\right)_{5}\left(\begin{array}{ll}
0 & s \\
j & s
\end{array}\right)_{5}\right.\right. \\
& \left.\left.+\left(\begin{array}{ll}
i & s \\
j & s
\end{array}\right)_{5}\left(\begin{array}{ll}
0 & s \\
0 & s
\end{array}\right)_{5}\right]\right\}_{4}^{s}+\sum_{s, t=1}^{5} \frac{1}{()_{5}\left(\begin{array}{l}
s \\
s
\end{array}\right)_{5}^{2}}\left(\begin{array}{l}
0 \\
k
\end{array}\right)_{5}\left\{\left(\begin{array}{l}
s \\
s
\end{array}\right)_{5}\left[\left(\begin{array}{ll}
0 & s \\
0 & j
\end{array}\right)_{5}\left(\begin{array}{ll}
t & s \\
i & s
\end{array}\right)_{5}-\left(\begin{array}{ll}
0 & i \\
s & j
\end{array}\right)_{5}\left(\begin{array}{ll}
t & s \\
0 & s
\end{array}\right)_{5}\right]\right. \\
& \left.+\left(\begin{array}{l}
s \\
0
\end{array}\right)_{5}\left[\left(\begin{array}{ll}
0 & s \\
j & s
\end{array}\right)_{5}\left(\begin{array}{ll}
t & s \\
i & s
\end{array}\right)_{5}+\left(\begin{array}{cc}
j & s \\
i & s
\end{array}\right)_{5}\left(\begin{array}{ll}
t & s \\
0 & s
\end{array}\right)_{5}\right]\right\} I_{3}^{s t} .
\end{aligned}
$$

Here the following "master formula" (Eq. (A.13) of [25]) is of great help:

$$
\begin{aligned}
\left(\begin{array}{l}
s \\
i
\end{array}\right)_{5}\left(\begin{array}{ll}
s & \tau \\
0 & s
\end{array}\right)_{5} & =\left(\begin{array}{l}
s \\
0
\end{array}\right)_{5}\left(\begin{array}{ll}
s & \tau \\
i & s
\end{array}\right)_{5}+\left(\begin{array}{l}
s \\
s
\end{array}\right)_{5}\left(\begin{array}{ll}
s & \tau \\
0 & i
\end{array}\right)_{5}, \\
\tau & =0,1, \ldots 5,
\end{aligned}
$$

which yields explicitly

$$
\left(\begin{array}{l}
s \\
s
\end{array}\right)_{5}\left(\begin{array}{ll}
0 & s \\
0 & i
\end{array}\right)_{5}+\left(\begin{array}{l}
s \\
0
\end{array}\right)_{5}\left(\begin{array}{ll}
0 & s \\
i & s
\end{array}\right)_{5}=\left(\begin{array}{l}
s \\
i
\end{array}\right)_{5}\left(\begin{array}{ll}
0 & s \\
0 & s
\end{array}\right)_{5},
$$

and

$$
\left(\begin{array}{l}
s \\
s
\end{array}\right)_{5}\left(\begin{array}{ll}
0 & j \\
s & i
\end{array}\right)_{5}-\left(\begin{array}{l}
s \\
0
\end{array}\right)_{5}\left(\begin{array}{ll}
i & s \\
j & s
\end{array}\right)_{5}=-\left(\begin{array}{l}
s \\
j
\end{array}\right)_{5}\left(\begin{array}{ll}
0 & s \\
i & s
\end{array}\right)_{5},
$$

so that (3.23) reads

$$
\begin{aligned}
A= & -\sum_{s=1}^{5} \frac{1}{()_{5}\left(\begin{array}{l}
s \\
s
\end{array}\right)_{5}^{2}}\left(\begin{array}{l}
0 \\
k
\end{array}\right)_{5} \cdot\left\{\left[\left(\begin{array}{l}
s \\
i
\end{array}\right)_{5}\left(\begin{array}{ll}
0 & s \\
j & s
\end{array}\right)_{5}\right.\right. \\
& \left.+\left(\begin{array}{l}
s \\
j
\end{array}\right)_{5}\left(\begin{array}{ll}
0 & s \\
i & s
\end{array}\right)_{5}\right]\left(\begin{array}{ll}
0 & s \\
0 & s
\end{array}\right)_{5} I_{4}^{s} \\
& -\sum_{t=1}^{5}\left[\left(\begin{array}{l}
s \\
j
\end{array}\right)_{5}\left(\begin{array}{ll}
0 & s \\
0 & s
\end{array}\right)_{5}\left(\begin{array}{ll}
t & s \\
i & s
\end{array}\right)_{5}\right. \\
& \left.\left.+\left(\begin{array}{c}
s \\
i
\end{array}\right)_{5}\left(\begin{array}{ll}
0 & s \\
j & s
\end{array}\right)_{5}\left(\begin{array}{ll}
t & s \\
0 & s
\end{array}\right)_{5}\right] I_{3}^{s t}\right\} .
\end{aligned}
$$

Next we will use

$$
\left(\begin{array}{l}
0 \\
k
\end{array}\right)_{5}\left(\begin{array}{l}
s \\
i
\end{array}\right)_{5}=-\left(\begin{array}{ll}
0 & i \\
s & k
\end{array}\right)_{5}()_{5}+\left(\begin{array}{l}
i \\
k
\end{array}\right)_{5}\left(\begin{array}{l}
s \\
0
\end{array}\right)_{5}
$$

As trivial as this relation may look, it plays the crucial role of splitting off

$$
\left(\begin{array}{l}
i \\
k
\end{array}\right)_{5}
$$

in order to produce $g^{\mu \nu}$ terms. It might also have been written as

$$
\left.\left(\begin{array}{l}
0 \\
k
\end{array}\right)_{5}\left(\begin{array}{l}
s \\
i
\end{array}\right)_{5}=\left(\begin{array}{ll}
0 & s \\
k & i
\end{array}\right)_{5}\right)_{5}+\left(\begin{array}{l}
0 \\
i
\end{array}\right)_{5}\left(\begin{array}{l}
s \\
k
\end{array}\right)_{5}
$$

but then it would not fulfill its purpose.

The first term at the right-hand side(r.h.s.) of (3.28) cancels a ()$_{5}$, while the second term enters the $g_{\mu \nu}$-terms, all of which are collected in (3.36). The complete coefficient of $I_{4}^{S}$ in (3.16) is thus given by

$$
\begin{aligned}
& S_{i j k}^{4, s}=\frac{1}{3\left(\begin{array}{l}
0 \\
0
\end{array}\right)_{5}\left(\begin{array}{l}
s \\
s
\end{array}\right)_{5}^{2}}\left\{-\left(\begin{array}{ll}
0 & s \\
0 & k
\end{array}\right)_{5}\left[\left(\begin{array}{ll}
0 & s \\
i & s
\end{array}\right)_{5}\left(\begin{array}{ll}
0 & s \\
j & s
\end{array}\right)_{5}+\left(\begin{array}{ll}
i & s \\
j & s
\end{array}\right)_{5}\left(\begin{array}{ll}
0 & s \\
0 & s
\end{array}\right)_{5}\right]\right. \\
& \left.+\left[\left(\begin{array}{ll}
0 & i \\
s & k
\end{array}\right)_{5}\left(\begin{array}{ll}
0 & s \\
j & s
\end{array}\right)_{5}+\left(\begin{array}{ll}
0 & j \\
s & k
\end{array}\right)_{5}\left(\begin{array}{ll}
0 & s \\
i & s
\end{array}\right)_{5}\right]\left(\begin{array}{ll}
0 & s \\
0 & s
\end{array}\right)_{5}+(i \leftrightarrow k)+(j \leftrightarrow k)\right\} .
\end{aligned}
$$


Finally, we have to investigate the last line of (3.22), being The left with the factor

$$
\left(\begin{array}{l}
0 \\
k
\end{array}\right)_{5}\left(\begin{array}{l}
s \\
0
\end{array}\right)_{5}
$$

as before in (3.21). The master formula (3.24) then yields $\left(\begin{array}{l}s \\ 0\end{array}\right)_{5}\left(\begin{array}{ll}t & s \\ j & s\end{array}\right)_{5}=\left(\begin{array}{l}s \\ j\end{array}\right)_{5}\left(\begin{array}{ll}t & s \\ 0 & s\end{array}\right)_{5}-\left(\begin{array}{l}s \\ s\end{array}\right)_{5}\left(\begin{array}{ll}t & s \\ 0 & j\end{array}\right)_{5}$.

$$
\left(\begin{array}{l}
s \\
s
\end{array}\right)_{5}
$$

in the second term of (3.31) cancels and the remaining factor is antisymmetric in $s$ and $t$, i.e. this term drops out after summation over $s, t$. Using again (3.28) and dropping for the time being the contribution to $g_{\mu \nu}$ terms, we finally write the coefficients of $I_{3}^{s t}$ and $I_{2}^{s t u}$ in the following way, taking care of the original $(i \leftrightarrow j)$ symmetry in (3.22):

$$
\begin{aligned}
& S_{i j k}^{3, s t}=\frac{1}{3\left(\begin{array}{l}
0 \\
0
\end{array}\right)\left(\begin{array}{l}
s \\
s
\end{array}\right)_{5}^{2}}\left\{\left(\begin{array}{ll}
0 & s \\
0 & k
\end{array}\right)_{5}\left[\left(\begin{array}{ll}
t & s \\
i & s
\end{array}\right)_{5}\left(\begin{array}{ll}
0 & s \\
j & s
\end{array}\right)_{5}+\left(\begin{array}{ll}
i & s \\
j & s
\end{array}\right)_{5}\left(\begin{array}{ll}
t & s \\
0 & s
\end{array}\right)_{5}+\frac{\left(\begin{array}{l}
s \\
s
\end{array}\right)_{5}\left(\begin{array}{lll}
0 & s & t \\
i & s & t
\end{array}\right)_{5}}{\left(\begin{array}{ll}
s & t \\
s & t
\end{array}\right)_{5}}\left(\begin{array}{ll}
s \\
j & s
\end{array}\right)_{5}\right]\right.
\end{aligned}
$$

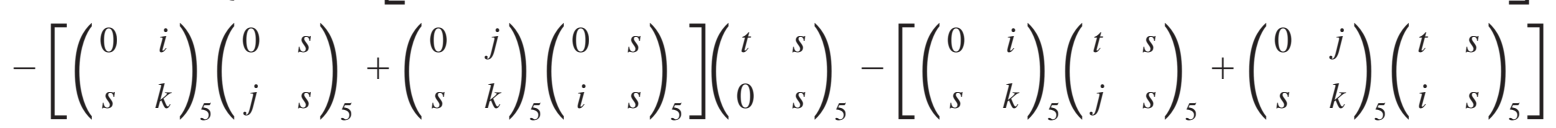

$$
\begin{aligned}
& \left.\times \frac{\left(\begin{array}{l}
s \\
s
\end{array}\right)_{5}\left(\begin{array}{lll}
0 & s & t \\
0 & s & t
\end{array}\right)_{5}}{2\left(\begin{array}{ll}
s & t \\
s & t
\end{array}\right)_{5}}+(i \leftrightarrow k)+(j \leftrightarrow k)\right\},
\end{aligned}
$$

and

$$
\begin{aligned}
S_{i j k}^{2, s t u}= & -\frac{1}{3\left(\begin{array}{l}
0 \\
0
\end{array}\right)_{5}\left(\begin{array}{l}
s \\
s
\end{array}\right)_{5}\left(\begin{array}{ll}
s & t \\
s & t
\end{array}\right)_{5}}\left\{\left(\begin{array}{ll}
0 & s \\
0 & k
\end{array}\right)_{5}\left(\begin{array}{ll}
t & s \\
j & s
\end{array}\right)_{5}\left(\begin{array}{lll}
u & s & t \\
i & s & t
\end{array}\right)_{5}\right. \\
& \left.-\frac{1}{2}\left[\left(\begin{array}{ll}
0 & j \\
s & k
\end{array}\right)_{5}\left(\begin{array}{lll}
u & s & t \\
i & s & t
\end{array}\right)_{5}+\left(\begin{array}{ll}
0 & i \\
s & k
\end{array}\right)_{5}\left(\begin{array}{lll}
u & s & t \\
j & s & t
\end{array}\right)_{5}\right]\left(\begin{array}{ll}
t & s \\
0 & s
\end{array}\right)_{5}+(i \leftrightarrow k)+(j \leftrightarrow k)\right\} .
\end{aligned}
$$

At the end we can determine the $g_{\mu \nu}$ terms from the above by collecting all terms containing factors of the type

$$
\left(\begin{array}{l}
i \\
j
\end{array}\right)_{5}
$$

$$
\begin{aligned}
\sum_{j=1}^{4} g^{[\mu \nu} q_{j}^{\lambda]} E_{00 j}= & \frac{2}{()_{5}} \sum_{i j k=1}^{4}\left[\left(\begin{array}{l}
j \\
k
\end{array}\right)_{5} E_{00 i}+\left(\begin{array}{l}
i \\
k
\end{array}\right)_{5} E_{00 j}\right. \\
& \left.+\left(\begin{array}{c}
i \\
j
\end{array}\right)_{5} E_{00 k}\right] q_{i}^{\mu} q_{j}^{\nu} q_{k}^{\lambda},
\end{aligned}
$$

where the square bracket means symmetrization of the included indices,

$$
g^{[\mu \nu} q_{k}^{\lambda]}=g^{\mu \nu} q_{k}^{\lambda}+g^{\mu \lambda} q_{k}^{\nu}+g^{\nu \lambda} q_{k}^{\mu},
$$

and use has been made of (3.6). Collecting all terms of type

$$
\left(\begin{array}{l}
i \\
j
\end{array}\right)_{5}
$$

in (3.22) we have 


$$
\begin{aligned}
& 3\left(\begin{array}{l}
0 \\
0
\end{array}\right)_{5} E_{00 j}=-\frac{1}{2} \sum_{s=1}^{5} \frac{1}{\left(\begin{array}{l}
s \\
s
\end{array}\right)_{5}^{2}}\left[2\left(\begin{array}{l}
s \\
0
\end{array}\right)_{5}\left(\begin{array}{ll}
0 & s \\
j & s
\end{array}\right)_{5}-\left(\begin{array}{l}
s \\
s
\end{array}\right)_{5}\left(\begin{array}{ll}
0 & s \\
0 & j
\end{array}\right)_{5}\right]\left(\begin{array}{ll}
0 & s \\
0 & s
\end{array}\right)_{5} I_{4}^{s} \\
& +\frac{1}{2} \sum_{s, t=1}^{5}\left\{\frac{1}{\left(\begin{array}{l}
s \\
s
\end{array}\right)_{5}^{2}}\left[\left(\begin{array}{l}
s \\
0
\end{array}\right)_{5}\left(\begin{array}{ll}
0 & s \\
j & s
\end{array}\right)_{5}-\left(\begin{array}{l}
s \\
s
\end{array}\right)_{5}\left(\begin{array}{ll}
0 & s \\
0 & j
\end{array}\right)_{5}\right]\left(\begin{array}{ll}
t & s \\
0 & s
\end{array}\right)_{5}+\frac{1}{\left(\begin{array}{l}
s \\
s
\end{array}\right)_{5}^{2}}\left(\begin{array}{l}
s \\
0
\end{array}\right)_{5}\left(\begin{array}{ll}
t & s \\
j & s
\end{array}\right)_{5}\left(\begin{array}{ll}
0 & s \\
0 & s
\end{array}\right)_{5}\right. \\
& +\frac{1}{\left(\begin{array}{l}
s \\
s
\end{array}\right)_{5}\left(\begin{array}{ll}
s & t \\
s & t
\end{array}\right)_{5}}\left(\begin{array}{l}
s \\
0
\end{array}\right)_{5}\left(\begin{array}{ll}
t & s \\
0 & s
\end{array}\right)_{5}\left(\begin{array}{lll}
0 & s & t \\
j & s & t
\end{array}\right)_{5} I_{3}^{s t} \\
& -\frac{1}{2} \sum_{s, t, u=1}^{5} \frac{1}{\left(\begin{array}{ll}
s \\
s
\end{array}\right)_{5}\left(\begin{array}{ll}
s & t \\
s & t
\end{array}\right)_{5}}\left(\begin{array}{l}
s \\
0
\end{array}\right)_{5}\left(\begin{array}{ll}
t & s \\
0 & s
\end{array}\right)_{5}\left(\begin{array}{lll}
u & s & t \\
j & s & t
\end{array}\right)_{5} I_{2}^{s t u} \text {. }
\end{aligned}
$$

The following relation can be proven by multiplication with

$$
\left(\begin{array}{l}
s \\
s
\end{array}\right)_{5}
$$

transforming it into the relation for an extensional of Eq. (A.8) of [25]:

$$
\left(\begin{array}{l}
s \\
0
\end{array}\right)_{5}\left(\begin{array}{ccc}
\mu & s & t \\
j & s & t
\end{array}\right)_{5}=\left(\begin{array}{l}
s \\
j
\end{array}\right)_{5}\left(\begin{array}{ccc}
\mu & s & t \\
0 & s & t
\end{array}\right)_{5}-\left(\begin{array}{cc}
\mu & s \\
0 & j
\end{array}\right)_{5}\left(\begin{array}{cc}
s & t \\
s & t
\end{array}\right)_{5}+\left(\begin{array}{cc}
t & s \\
0 & j
\end{array}\right)_{5}\left(\begin{array}{cc}
t & s \\
\mu & s
\end{array}\right)_{5} \quad \mu=0,1, \cdots, 4 .
$$

It turns out to be useful for the simplification of the coefficients of $I_{3}^{s t}$ and $I_{2}^{s t u}$ in (3.36). For the coefficient of $I_{3}^{s t}$, we apply relation (3.37) with $\mu=0$. The last term on the r.h.s. of (3.37) is combined with the last term on the second line of (3.36) using (3.31):

$$
\begin{aligned}
& \frac{1}{\left(\begin{array}{l}
s \\
s
\end{array}\right)_{5}^{2}}\left\{\frac{\left(\begin{array}{l}
s \\
s
\end{array}\right)_{5}}{\left(\begin{array}{ll}
s & t \\
s & t
\end{array}\right)_{5}}\left(\begin{array}{ll}
t & s \\
0 & j
\end{array}\right)_{5}\left(\begin{array}{ll}
t & s \\
0 & s
\end{array}\right)_{5}^{2}+\left(\begin{array}{l}
s \\
0
\end{array}\right)_{5}\left(\begin{array}{ll}
t & s \\
j & s
\end{array}\right)_{5}\left(\begin{array}{ll}
0 & s \\
0 & s
\end{array}\right)_{5}\right\} \\
& =\frac{1}{\left(\begin{array}{l}
s \\
s
\end{array}\right)_{5}^{2}}\left(\begin{array}{l}
s \\
j
\end{array}\right)_{5}\left(\begin{array}{ll}
t & s \\
0 & s
\end{array}\right)_{5}\left(\begin{array}{ll}
0 & s \\
0 & s
\end{array}\right)_{5}-\frac{\left(\begin{array}{ll}
t & s \\
0 & j
\end{array}\right)_{5}\left(\begin{array}{lll}
0 & s & t \\
0 & s & t
\end{array}\right)_{5}}{\left(\begin{array}{ll}
s & t \\
s & t
\end{array}\right)_{5} .},
\end{aligned}
$$

After summation over $s$ and $t$, the last term on the r.h.s. will vanish. Furthermore, we apply (3.24) taking $\tau=0$.

For the coefficient of $I_{2}^{s t u}$ in (3.36) we apply relation (3.37) with $\mu=u$. Since $I_{2}^{s t u}$ is symmetric in $s, t$, and $u$, we consider the sum over all permutations of any fixed set of values of $s, t$, and $u$. We find that

$$
\sum_{\text {permutations }} \frac{1}{\left(\begin{array}{l}
s \\
s
\end{array}\right)_{5}\left(\begin{array}{ll}
s & t \\
s & t
\end{array}\right)_{5}}\left(\begin{array}{cc}
t & s \\
0 & s
\end{array}\right)_{5}\left[\left(\begin{array}{cc}
u & s \\
0 & j
\end{array}\right)_{5}\left(\begin{array}{ll}
s & t \\
s & t
\end{array}\right)_{5}-\left(\begin{array}{cc}
t & s \\
0 & j
\end{array}\right)_{5}\left(\begin{array}{cc}
t & s \\
u & s
\end{array}\right)_{5}\right]=0
$$

so that the two last terms on the r.h.s. of (3.37) can be dropped in this case. Thus we have 


$$
\begin{aligned}
& 3\left(\begin{array}{c}
0 \\
0
\end{array}\right)_{5} E_{00 j}=-\frac{1}{2} \sum_{s=1}^{5} \frac{1}{\left(\begin{array}{l}
s \\
s
\end{array}\right)_{5}^{2}}\left[3\left(\begin{array}{c}
s \\
0
\end{array}\right)_{5}\left(\begin{array}{cc}
0 & s \\
j & s
\end{array}\right)_{5}-\left(\begin{array}{c}
s \\
j
\end{array}\right)_{5}\left(\begin{array}{cc}
0 & s \\
0 & s
\end{array}\right)_{5}\right]\left(\begin{array}{ll}
0 & s \\
0 & s
\end{array}\right)_{5} I_{4}^{s}+\frac{1}{2} \sum_{s, t=1}^{5} \frac{1}{\left(\begin{array}{l}
s \\
s
\end{array}\right)_{5}^{2}}\left[3\left(\begin{array}{l}
s \\
0
\end{array}\right)_{5}\left(\begin{array}{ll}
0 & s \\
j & s
\end{array}\right)_{5}\right. \\
& \left.-\left(\begin{array}{l}
s \\
j
\end{array}\right)_{5} \frac{\left(\begin{array}{ll}
t & s \\
0 & s
\end{array}\right)_{5}}{\left(\begin{array}{ll}
s & t \\
s & t
\end{array}\right)_{5}}\right]\left(\begin{array}{cc}
t & s \\
0 & s
\end{array}\right)_{5} I_{3}^{s t}-\frac{1}{2} \sum_{s, t, u=1}^{5} \frac{1}{\left(\begin{array}{l}
s \\
s
\end{array}\right)_{5}^{2}}\left(\begin{array}{l}
s \\
j
\end{array}\right)_{5} \frac{\left(\begin{array}{c}
s \\
s
\end{array}\right)_{5}\left(\begin{array}{ccc}
u & s & t \\
0 & s & t
\end{array}\right)_{5}}{\left(\begin{array}{ll}
s & t \\
s & t
\end{array}\right)_{5}}\left(\begin{array}{ll}
t & s \\
0 & s
\end{array}\right)_{5} I_{2}^{s t u} .
\end{aligned}
$$

Collecting all contributions, our final result for the tensor of rank 3 can be written as

$$
\begin{gathered}
I_{5}^{\mu \nu \lambda}=\sum_{i, j, k=1}^{4} q_{i}^{\mu} q_{j}^{\nu} q_{k}^{\lambda} E_{i j k}+\sum_{k=1}^{4} g^{[\mu \nu} q_{k}^{\lambda]} E_{00 k}, \\
E_{i j k}=\sum_{s=1}^{5} S_{i j k}^{4, s} I_{4}^{s}+\sum_{s, t=1}^{5} S_{i j k}^{3, s t} I_{3}^{s t}+\sum_{s, t, u=1}^{5} S_{i j k}^{2, s t u} I_{2}^{s t u},
\end{gathered}
$$

and the coefficients $S_{i j k}^{4, s}, S_{i j k}^{3, s t}, S_{i j k}^{2, s t u}$ are given in (3.30), (3.32), and (3.33) and $E_{00 k}$ in (3.40).

\section{HEXAGONS}

The 6-point function has the nice property that the tensors of rank $R$ can be reduced to a sum of six 5-point tensors of rank $R-1$. This property has also been derived in [5]; an earlier demonstration of this property, however, has been given already in [29]. The simplification in this case is due to the fact that ()$_{6} \equiv 0$, which has extensively been discussed in [29]. Beyond that, in our approach, the above results for the 5-point tensors can be directly used, thus reducing the 6-point tensors of up to rank $R=4$ to scalar 4- and 3- and 2-point integrals. Particularly simple results are thus obtained for the 6-point tensors using the results of Appendix A and Sections III A and III B. What was missing in [29] is exactly this simplification, which comes with the cancellation of the Gram determinant ()$_{5}$; see Appendix A of that paper.

\section{A. Scalar and vector integrals}

According to (I.33) we write (see [25] and also (I.55))

$$
I_{6}=\sum_{r=1}^{6} \frac{\left(\begin{array}{l}
0 \\
r
\end{array}\right)_{6}}{\left(\begin{array}{l}
0 \\
0
\end{array}\right)_{6}} E^{r}=\sum_{r=1}^{6} \frac{\left(\begin{array}{l}
r \\
k
\end{array}\right)_{6}}{\left(\begin{array}{l}
0 \\
k
\end{array}\right)_{6}} E^{r}, \quad k=1, \ldots, 6,
$$

and (3.2) now reads

$$
E^{r} \equiv I_{5}^{r}=\frac{1}{\left(\begin{array}{ll}
0 & r \\
0 & r
\end{array}\right)_{6}} \sum_{s=1, s \neq r}^{6}\left(\begin{array}{ll}
0 & r \\
s & r
\end{array}\right)_{6} I_{4}^{r s} .
$$

Here we see already the general scheme of reducing 6- point functions to 5-point functions: In general, in any signed minor $(\cdots)_{5}$ a further column

$$
\left(\begin{array}{l}
r \\
r
\end{array}\right)
$$

is scratched, resulting in a $(\cdots)_{6}$ and in the scalar functions a further propagator is scratched.

As in (3.3) and (3.4), with the use of (I.57), we obtain

$$
I_{6}^{\mu}=\sum_{i=1}^{5} q_{i}^{\mu} I_{6, i}
$$

$$
\begin{aligned}
I_{6, i} & =-I_{6, i}^{[d+]} \\
& =(d-5) \frac{\left(\begin{array}{l}
0 \\
i
\end{array}\right)_{6}}{\left(\begin{array}{l}
0 \\
0
\end{array}\right)_{6}} I_{6}^{[d+]}-\frac{1}{\left(\begin{array}{l}
0 \\
0
\end{array}\right)_{6}} \sum_{r=1}^{6}\left(\begin{array}{ll}
0 & i \\
0 & r
\end{array}\right)_{6} I_{5}^{r} .
\end{aligned}
$$

While in (3.4) the first part vanishes in the limit $d \rightarrow 4$, here its disappearance is due to (I.61):

$$
\sum_{i=1}^{5} q_{i}^{\mu}\left(\begin{array}{l}
0 \\
i
\end{array}\right)_{6}=0
$$

Indeed (4.5) will play a crucial role for the higher tensor reduction. The resulting form in (4.4) is already the generic form for the higher tensors too! Therefore, it appears useful to introduce the vector, applying further (A.15) of [25] and (I.61):

$$
\begin{aligned}
v_{r}^{\mu} & =-\frac{1}{\left(\begin{array}{l}
0 \\
0
\end{array}\right)_{6}} \sum_{i=1}^{5}\left(\begin{array}{ll}
0 & i \\
0 & r
\end{array}\right)_{6} q_{i}^{\mu} \\
& =-\frac{1}{\left(\begin{array}{l}
0 \\
k
\end{array}\right)_{6}} \sum_{i=1}^{5}\left(\begin{array}{ll}
0 & r \\
k & i
\end{array}\right)_{6} q_{i}^{\mu}, \quad k=0, \ldots, 6
\end{aligned}
$$

summing over all 5 (dependent) vectors. $v_{r}$ projected on these vectors reads 


$$
\begin{aligned}
v_{r} \cdot q_{i}=-\frac{1}{2}\left[\delta_{i r}-\left(Y_{i 6}-Y_{66}\right) \frac{\left(\begin{array}{l}
0 \\
r
\end{array}\right)_{6}}{\left(\begin{array}{l}
0 \\
0
\end{array}\right)_{6}}\right] \\
=-\frac{1}{2}\left[\delta_{i r}+\left(q_{i}^{2}+m_{6}^{2}-m_{i}^{2}\right) \frac{\left(\begin{array}{l}
0 \\
r)_{6} \\
\left(\begin{array}{l}
0 \\
0
\end{array}\right)_{6}
\end{array}\right] .}{} .\right.
\end{aligned}
$$

With this definition we can write in a compact way

$$
I_{6}^{\mu}=\sum_{r=1}^{6} v_{r}^{\mu} E^{r}
$$

\section{B. $\boldsymbol{R}=\mathbf{2}$ tensor integrals}

The Eq. (2.2) reads in this case

$$
I_{6}^{\mu \nu}=\sum_{i, j=1}^{5} q_{i}^{\mu} q_{j}^{\nu} \nu_{i j} I_{6, i j}^{[d+]^{2}}-\frac{1}{2} g^{\mu \nu} I_{6}^{[d+]},
$$

and by using (I.59) we have

$$
\begin{aligned}
& \nu_{i j} I_{6, i j}^{[d+]^{2}}=-(d-4) \frac{\left(\begin{array}{l}
0 \\
i
\end{array}\right)_{6}}{\left(\begin{array}{l}
0 \\
0
\end{array}\right)_{6}^{[d+]^{2}}} I_{6, i}+\frac{\left(\begin{array}{ll}
0 & i \\
0 & j
\end{array}\right)_{6}}{\left(\begin{array}{l}
0 \\
0
\end{array}\right)_{6}} I_{6}^{[d+]} \\
& +\frac{1}{\left(\begin{array}{l}
0 \\
0
\end{array}\right)_{6}} \sum_{r=1, r \neq i}^{6}\left(\begin{array}{ll}
0 & j \\
0 & r
\end{array}\right)_{6} I_{5, i}^{[d+], r} \text {. }
\end{aligned}
$$

We consider the limit $d \rightarrow 4$ and use (I.67):

$$
g^{\mu \nu}=\frac{2}{\left(\begin{array}{l}
0 \\
0
\end{array}\right)_{6}} \sum_{i, j=1}^{5}\left(\begin{array}{ll}
0 & i \\
0 & j
\end{array}\right)_{6} q_{i}^{\mu} q_{j}^{\nu} .
$$

Writing it like in (3.5),

$$
I_{6}^{\mu \nu}=\sum_{i, j=1}^{5} q_{i}^{\mu} q_{j}^{\nu} I_{6, i j}
$$

we obtain by using (3.4)

$$
I_{6, i j}=-\frac{1}{\left(\begin{array}{l}
0 \\
0
\end{array}\right)_{6}} \sum_{r=1, r \neq i}^{6}\left(\begin{array}{ll}
0 & j \\
0 & r
\end{array}\right)_{6} E_{i}^{r}
$$

to be compared with (4.4). For completeness we specify $E_{i}^{r}$, which we read off from (3.4) to be

$$
E_{i}^{r}=-\frac{1}{\left(\begin{array}{ll}
0 & r \\
0 & r
\end{array}\right)_{6}} \sum_{s=1}^{6}\left(\begin{array}{lll}
0 & i & r \\
0 & s & r
\end{array}\right)_{6} I_{4}^{r s},
$$

and finally

$$
I_{6}^{\mu \nu}=\sum_{i=1}^{5} q_{i}^{\mu} \sum_{r=1, r \neq i}^{6} v_{r}^{\nu} E_{i}^{r}
$$

We remark that due to (4.14), $E_{i}^{r}=0$ for $r=i$ and correspondingly this will be the case for all higher tensors such that limitations like $r \neq i$ could be dropped but are convenient to keep in numerical programs.

\section{C. $\boldsymbol{R}=\mathbf{3}$ tensor integrals}

Equation (2.3) reads in this case

$$
\begin{aligned}
I_{6}^{\mu \nu \lambda}= & -\sum_{i, j, k=1}^{5} q_{i}^{\mu} q_{j}^{\nu} q_{k}^{\lambda} \nu_{i j} \nu_{i j k} I_{6, i j k}^{[d+]^{3}} \\
& +\frac{1}{2} \sum_{i=1}^{5}\left(g^{\mu \nu} q_{i}^{\lambda}+g^{\mu \lambda} q_{i}^{\nu}+g^{\nu \lambda} q_{i}^{\mu}\right) I_{6, i}^{[d+]^{2}},
\end{aligned}
$$

and with (I.60) we have

$$
\begin{aligned}
& \nu_{i j} \nu_{i j k} I_{6, i j k}^{[d+]^{3}}=-(d-3) \frac{\left(\begin{array}{l}
0 \\
k
\end{array}\right)_{6}}{\left(\begin{array}{l}
0 \\
0
\end{array}\right)_{6}^{[d+]^{2}}} I_{6, i j}+\frac{\left(\begin{array}{ll}
0 & k \\
0 & i
\end{array}\right)_{6}}{\left(\begin{array}{l}
0 \\
0
\end{array}\right)_{6}} I_{6, j}^{[d+]^{2}} \\
& +\frac{\left(\begin{array}{ll}
0 & k \\
0 & j
\end{array}\right)_{6}}{\left(\begin{array}{l}
0 \\
0
\end{array}\right)_{6}^{[d+]^{2}}} \\
& +\frac{1}{\left(\begin{array}{l}
0 \\
0
\end{array}\right)_{6}} \sum_{r=1, r \neq i, j}^{6}\left(\begin{array}{ll}
0 & k \\
0 & r
\end{array}\right)_{6} \nu_{i j} I_{5, i j}^{[d+]^{2}, r} \text {. }
\end{aligned}
$$

The first term on the r.h.s. is eliminated due to (4.5) and the next two terms cancel due to (4.11). Taking into account $I_{5}^{[d+]}$, relation (3.7) now reads

$$
I_{5, i j}^{r}=\nu_{i j} I_{5, i j}^{[d+]^{2}, r}-\frac{\left(\begin{array}{cc}
i & r \\
j & r
\end{array}\right)_{6}}{\left(\begin{array}{l}
r \\
r
\end{array}\right)_{6}} I_{5}^{[d+], r} .
$$

As a further representation of $g^{\mu \nu}$ we have [see (I.75)]

$$
g^{\mu \nu}=\frac{2}{\left(\begin{array}{l}
r \\
r
\end{array}\right)_{6}} \sum_{i, j=1}^{5}\left(\begin{array}{ll}
i & r \\
j & r
\end{array}\right)_{6} q_{i}^{\mu} q_{j}^{\nu}, \quad r=1 \ldots 6 .
$$

Using again (I.57) and the definition 


$$
I_{6}^{\mu \nu \lambda}=\sum_{i, j, k=1}^{5} q_{i}^{\mu} q_{j}^{\nu} q_{k}^{\lambda} I_{6, i j k}
$$

we obtain

$$
I_{6, i j k}=-\frac{1}{\left(\begin{array}{l}
0 \\
0
\end{array}\right)_{6}} \sum_{r=1, r \neq i, j}^{6}\left(\begin{array}{ll}
0 & k \\
0 & r
\end{array}\right)_{6} I_{5, i j}^{r} .
$$

From (3.10) and (4.19) $I_{5, i j}^{r}$ reads

$$
I_{5, i j}^{r}=E_{i j}^{r}+2 \frac{\left(\begin{array}{ll}
i & r \\
j & r
\end{array}\right)_{6}}{\left(\begin{array}{l}
r \\
r
\end{array}\right)_{6}} E_{00}^{r},
$$

so that we get

$$
\begin{aligned}
I_{6}^{\mu \nu \lambda}= & \sum_{i, j=1}^{5} q_{i}^{\mu} q_{j}^{\nu} \sum_{r=1, r \neq i, j}^{6} v_{r}^{\lambda} E_{i j}^{r} \\
& +\sum_{i, j=1}^{5} q_{i}^{\mu} q_{j}^{\nu} \sum_{r=1}^{6} 2 \frac{\left(\begin{array}{ll}
i & r \\
j & r
\end{array}\right)_{6}}{\left(\begin{array}{l}
r \\
r
\end{array}\right)_{6}} v_{r}^{\lambda} E_{00}^{r},
\end{aligned}
$$

where in the second term we can drop the limitation $r \neq$ $i, j$ since it is automatically fulfilled due to the numerator

$$
\left(\begin{array}{ll}
i & r \\
j & r
\end{array}\right) 6
$$

vanishing for $r=i$ and $r=j$. Thus summation over $i$ and $j$ is possible, using (4.19), with a result

$$
I_{6}^{\mu \nu \lambda}=\sum_{i, j=1}^{5} q_{i}^{\mu} q_{j}^{\nu} \sum_{r=1, r \neq i, j}^{6} v_{r}^{\lambda} E_{i j}^{r}+g^{\mu \nu} \sum_{r=1}^{6} v_{r}^{\lambda} E_{00}^{r}
$$

or

$$
I_{6}^{\mu \nu \lambda}=\sum_{r=1}^{6} v_{r}^{\lambda} I_{5}^{\mu \nu, r}
$$

with

$$
\begin{gathered}
I_{5}^{\mu \nu, r}=\sum_{i, j=1, i, j \neq r}^{5} q_{i}^{\mu} q_{j}^{\nu} E_{i j}^{r}+g^{\mu \nu} E_{00}^{r} . \\
\text { D. } \boldsymbol{R}=\mathbf{4} \text { tensor integrals }
\end{gathered}
$$

The tensor integral in (2.4) contains three different integrals in higher dimension, which have to be reduced or to be eliminated. We begin with $I_{n, i j k l}^{[d+]^{4}}$ using (I.26). For convenience we use $x$ instead of 4 :

$$
\begin{aligned}
\left(\begin{array}{l}
0 \\
0
\end{array}\right)_{n} \nu_{i j k l} \boldsymbol{l}^{+} I_{n, i j k}^{[d+]^{x}} \equiv & \left(\begin{array}{l}
0 \\
0
\end{array}\right)_{n} \nu_{i j k l} I_{n, i j k l}^{[d+]^{x}} \\
= & \sum_{r=1}^{n}\left(\begin{array}{ll}
0 & l \\
0 & r
\end{array}\right)_{n}[d+2 x-(n+3)] I_{n, i j k}^{[d+]^{x}}-\sum_{s=1}^{n}\left(\begin{array}{ll}
0 & l \\
0 & s
\end{array}\right)_{n} \nu_{i j k s} I_{n, i j k}^{[d+]^{x}}-\sum_{r, s=1 ; r \neq s}^{n}\left(\begin{array}{ll}
0 & l \\
0 & r
\end{array}\right)_{n} \nu_{i j k s} \mathbf{r}^{-} \mathbf{s}^{+} I_{n, i j k}^{[d+]^{x}} \\
= & \left\{[n+4-(d+2 x)]\left(\begin{array}{l}
0 \\
l
\end{array}\right)_{n}-\left(\begin{array}{ll}
0 & l \\
0 & i
\end{array}\right)_{n}-\left(\begin{array}{ll}
0 & l \\
0 & j
\end{array}\right)_{n}-\left(\begin{array}{ll}
0 & l \\
0 & k
\end{array}\right)_{n}\right\}_{n}^{[d+i j k} I_{n, i j k}^{x} \\
& -\sum_{r=1}^{n}\left(\begin{array}{ll}
0 & l \\
0 & r
\end{array}\right)_{n} \sum_{s=1 ; s \neq r}^{n} \nu_{i j k s} I_{n, i j k s}^{[d+]^{x}, r} .
\end{aligned}
$$

The last double sum in (4.27), assuming all indices $i, j, k$ to be different, reads

$$
\begin{aligned}
& -\left(\begin{array}{ll}
0 & l \\
0 & i
\end{array}\right)_{n} \sum_{s=1 ; s \neq i}^{n} \nu_{j k s} I_{n, j k s}^{[d+]^{x}}-\left(\begin{array}{ll}
0 & l \\
0 & j
\end{array}\right)_{n} \sum_{s=1 ; s \neq j}^{n} \nu_{i k s} I_{n, i k s}^{[d+]^{x}}-\left(\begin{array}{ll}
0 & l \\
0 & k
\end{array}\right) \sum_{n} \sum_{s=1 ; s \neq k}^{n} \nu_{i j s} I_{n, i j s}^{[d+]^{x}} \\
& -\sum_{r=1 ; r \neq i, j, k}^{n}\left(\begin{array}{ll}
0 & l \\
0 & r
\end{array}\right)_{n} \sum_{s=1 ; s \neq r}^{n} \nu_{i j k s} I_{n-1, i j k s}^{[d+]^{x}, r} .
\end{aligned}
$$

Now adding corresponding terms in (4.27) and (4.28), e.g. for $r=i$, we get

$$
-\left(\begin{array}{ll}
0 & l \\
0 & i
\end{array}\right)_{n} \sum_{s=1 ; s \neq i}^{n} \nu_{j k s} I_{n, j k s}^{[d+]^{x}}-\left(\begin{array}{cc}
0 & l \\
0 & i
\end{array}\right)_{n} I_{n, i j k}^{[d+]^{x}}=-\left(\begin{array}{cc}
0 & l \\
0 & i
\end{array}\right)_{n} \sum_{s=1}^{n} \nu_{j k s} I_{n, j k s}^{[d+]^{x}}=\left(\begin{array}{cc}
0 & l \\
0 & i
\end{array}\right)_{n} I_{n, j k}^{[d+]^{[x-1)}},
$$

due to (I.29). In case two indices are equal, e.g. $i=j \neq k$, we have 


$$
\begin{aligned}
-\left(\begin{array}{ll}
0 & l \\
0 & i
\end{array}\right)_{n} \sum_{s=1 ; s \neq i}^{n}\left(1+2 \delta_{i s}+\delta_{k s}\right) I_{n, i k s}^{[d+]^{x}}-2\left(\begin{array}{cc}
0 & l \\
0 & i
\end{array}\right)_{n} I_{n, i i s}^{[d+]^{x}} & =-\left(\begin{array}{ll}
0 & l \\
0 & i
\end{array}\right)_{n} \sum_{s=1}^{n}\left(1+\delta_{i s}+\delta_{k s}\right) I_{n, i k s}^{[d+]^{x}} \\
& \equiv-\left(\begin{array}{ll}
0 & l \\
0 & i
\end{array}\right)_{n} \sum_{s=1}^{n} \nu_{i k s} I_{n, i k s}^{[d+]^{x}},
\end{aligned}
$$

like (4.29), i.e. if two indices agree, this integral occurs only once. As final result we have

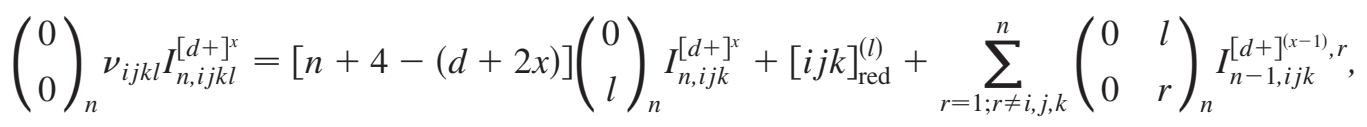

where according to (4.29) and the discussion thereafter

$$
[i j k]^{(l)}=\left(\begin{array}{ll}
0 & l \\
0 & i
\end{array}\right)_{n} I_{n, j k}^{[d+]^{(x-1)}}+\left(\begin{array}{cc}
0 & l \\
0 & j
\end{array}\right)_{n} I_{n, i k}^{[d+]^{(x-1)}}+\left(\begin{array}{ll}
0 & l \\
0 & k
\end{array}\right)_{n} I_{n, i j}^{[d+]^{(x-1)}}
$$

and $[i j k]_{\mathrm{red}}^{(l)}=[i j k]^{(l)}$ without repetition, e.g.

$$
[i i i]_{\mathrm{red}}^{(l)}=\left(\begin{array}{cc}
0 & l \\
0 & i
\end{array}\right)_{n} I_{n, i i}^{[d+]^{(x-1)}}
$$

Now, making use of $n_{i j k l}=\nu_{i j} \nu_{i j k} \nu_{i j k l}$, we see that due to (4.5) the first part in (4.31) drops out after insertion into (2.4). The second contribution of (4.31) yields

$$
\frac{1}{\left(\begin{array}{l}
0 \\
0
\end{array}\right)} \sum_{n}^{n-1} q_{l}^{\rho} \sum_{i, j, k=1}^{n-1} \nu_{i j} \nu_{i j k}[i j k]_{\mathrm{red}}^{(l)} q_{i}^{\mu} q_{j}^{\nu} q_{k}^{\lambda}
$$

We have

$$
\nu_{i j} \nu_{i j k}[i j k]_{\mathrm{red}}^{(l)}=[i j k]^{(l)}+\left(\begin{array}{cc}
0 & l \\
0 & i
\end{array}\right)_{n} \delta_{j k} I_{n, j k}^{[d+]^{(x-1)}}+\left(\begin{array}{ll}
0 & l \\
0 & j
\end{array}\right)_{n} \delta_{i k} I_{n, i k}^{[d+]^{(x-1)}}+\left(\begin{array}{ll}
0 & l \\
0 & k
\end{array}\right)_{n} \delta_{i j} I_{n, i j}^{[d+]^{(x-1)}},
$$

with the help of which (4.33) reads

$$
\begin{aligned}
& \frac{1}{\left(\begin{array}{l}
0 \\
0
\end{array}\right)_{n}} \sum_{l=1}^{n-1} q_{l}^{\rho} \sum_{i, j, k=1}^{n-1}\left[q_{i}^{\mu}\left(\begin{array}{ll}
0 & l \\
0 & i
\end{array}\right)_{n}\left(1+\delta_{j k}\right) I_{n, j k}^{[d+]^{(x-1)}} q_{j}^{\nu} q_{k}^{\lambda}+q_{j}^{\nu}\left(\begin{array}{ll}
0 & l \\
0 & j
\end{array}\right)_{n}\left(1+\delta_{i k}\right) I_{n, i k}^{[d+]^{(x-1)}} q_{i}^{\mu} q_{k}^{\lambda}\right. \\
& \left.\quad+q_{k}^{\lambda}\left(\begin{array}{ll}
0 & l \\
0 & k
\end{array}\right)_{n}\left(1+\delta_{i j}\right) I_{n, i j}^{[d+]^{(x-1)}} q_{i}^{\mu} q_{j}^{\nu}\right] .
\end{aligned}
$$

Using (I.67) we have for $d=4$

$$
\frac{1}{2}\left\{g^{\mu \rho} n_{j k} I_{n, j k}^{[d+]^{(x-1)}} q_{j}^{\nu} q_{k}^{\lambda}+g^{\nu \rho} n_{i k} I_{n, i k}^{[d+]^{(x-1)}} q_{i}^{\mu} q_{k}^{\lambda}+g^{\lambda \rho} n_{i j} I_{n, i j}^{[d+]^{(x-1)}} q_{i}^{\mu} q_{j}^{\nu}\right\}
$$

and we see that this contribution is canceled by the last three terms of the type $I_{n, j k}^{[d+]^{(x-1)}}$ in (2.4). The first three terms of this type are evaluated by means of (I.59) to yield

$$
n_{i j} I_{n, j k}^{[d+]^{(x-1)}}=\frac{1}{\left(\begin{array}{l}
0 \\
0
\end{array}\right)_{n}}\left\{[n+2(2-x)-d]\left(\begin{array}{l}
0 \\
j
\end{array}\right)_{n} I_{n, i}^{[d+]^{(x-1)}}+\left(\begin{array}{ll}
0 & i \\
0 & j
\end{array}\right)_{n} I_{n}^{[d+]^{(x-2)}}+\sum_{r=1 ; r \neq i}^{n}\left(\begin{array}{ll}
0 & j \\
0 & r
\end{array}\right)_{n} I_{n-1, i}^{[d+]^{(x-2), r}}\right\} .
$$

Inserting this into (2.4), the first part yields a vanishing contribution due to (4.5). The second term yields, again due to (4.5),

$$
-\frac{1}{2\left(\begin{array}{l}
0 \\
0
\end{array}\right)} \sum_{n}^{n-1}\left\{g^{\mu \nu} q_{i}^{\lambda} q_{j}^{\rho}+g^{\mu \lambda} q_{i}^{\nu} q_{j}^{\rho}+g^{\nu \lambda} q_{i}^{\mu} q_{j}^{\rho}\right\}\left(\begin{array}{ll}
0 & i \\
0 & j
\end{array}\right)_{n} I_{n}^{[d+]^{(x-2)}}=-\frac{1}{4}\left(g^{\mu \nu} g^{\lambda \rho}+g^{\mu \lambda} g^{\nu \rho}+g^{\mu \rho} g^{\nu \lambda}\right) I_{n}^{[d+]^{(x-2)}},
$$


which cancels the last term in (2.4) and the total contribution thus reads

$$
\begin{aligned}
& \frac{1}{\left(\begin{array}{l}
0 \\
0
\end{array}\right)} \int_{n} \sum_{i, j, k, l=1}^{n-1} \nu_{i j} \nu_{i j k} q_{i}^{\mu} q_{j}^{\nu} q_{k}^{\lambda} q_{l}^{\rho} \sum_{r=1 ; r \neq i, j, k}^{n}\left(\begin{array}{ll}
0 & l \\
0 & r
\end{array}\right)_{n} I_{n-1, i j k}^{[d+]^{(x-1)}, r}-\frac{1}{2} \sum_{i, j=1}^{n-1}\left(g^{\mu \nu} q_{i}^{\lambda} q_{j}^{\rho}+g^{\mu \lambda} q_{i}^{\nu} q_{j}^{\rho}+g^{\nu \lambda} q_{i}^{\mu} q_{j}^{\rho}\right) \\
& \left.\times \sum_{r=1 ; r \neq i}^{n}\left(\begin{array}{ll}
0 & j \\
0 & r
\end{array}\right)_{n} I_{n-1, i}^{[d+]^{(x-2),}, r}\right\},
\end{aligned}
$$

reducing the 6-point tensor to 5-point tensors in lower dimensions. For further reduction we put explicitly $n=6$ and $x=4$ and write (3.18) in the form

$$
\nu_{i j} \nu_{i j k} I_{5, i j k}^{[d+]^{3}, r}=-I_{5, i j k}^{r}+\left[\frac{\left(\begin{array}{ll}
j & r \\
k & r
\end{array}\right)_{6}}{\left(\begin{array}{l}
r \\
r
\end{array}\right)_{6}} I_{5, i}^{[d+]^{2}, r}+\frac{\left(\begin{array}{ll}
i & r \\
k & r
\end{array}\right)_{6}}{\left(\begin{array}{l}
r \\
r
\end{array}\right)_{6}} I_{5, j}^{[d+]^{2}, r}+\frac{\left(\begin{array}{ll}
i & r \\
j & r
\end{array}\right)_{6}}{\left(\begin{array}{l}
r \\
r
\end{array}\right)_{6}} I_{5, k}^{[d+]^{2}, r}\right] .
$$

With (4.19) it is now easy to see that the square bracket in (4.40) cancels out the second part in (4.39) and using the definition

$$
I_{6}^{\mu \nu \lambda \rho}=\sum_{i, j, k, l=1}^{5} q_{i}^{\mu} q_{j}^{\nu} q_{k}^{\lambda} q_{l}^{\rho} I_{6, i j k l}
$$

we obtain

$$
I_{6, i j k l}=-\frac{1}{\left(\begin{array}{l}
0 \\
0
\end{array}\right)_{6}} \sum_{r=1, r \neq i, j, k}^{6}\left(\begin{array}{ll}
0 & l \\
0 & r
\end{array}\right)_{6} I_{5, i j k}^{r} .
$$

Again, with (3.17) and (4.19) $I_{5, i j k}^{r}$ reads

$$
I_{5, i j k}^{r}=E_{i j k}^{r}+2 \frac{\left(\begin{array}{ll}
i & r \\
j & r
\end{array}\right)_{6}}{\left(\begin{array}{l}
r \\
r
\end{array}\right)_{6}} E_{00 k}^{r}+2 \frac{\left(\begin{array}{cc}
i & r \\
k & r
\end{array}\right)_{6}}{\left(\begin{array}{l}
r \\
r
\end{array}\right)_{6}} E_{00 j}^{r}+2 \frac{\left(\begin{array}{ll}
j & r \\
k & r
\end{array}\right)_{6}}{\left(\begin{array}{l}
r \\
r
\end{array}\right)_{6}} E_{00 i}^{r},
$$

so

$$
\begin{aligned}
& I_{6}^{\mu \nu \lambda \rho}=\sum_{i, j, k=1}^{5} q_{i}^{\mu} q_{j}^{\nu} q_{k}^{\lambda} \sum_{r=1, r \neq i, j, k}^{6} v_{r}^{\rho} E_{i j k}^{r}+\sum_{i, j, k=1}^{5} q_{i}^{\mu} q_{j}^{\nu} q_{k}^{\lambda} \sum_{r=1, r \neq i, j, k}^{6} v_{r}^{\rho}\left\{2 \frac{\left(\begin{array}{ll}
i & r \\
j & r
\end{array}\right)_{6}}{\left(\begin{array}{l}
r \\
r
\end{array}\right)_{6}} E_{00 k}^{r}+2 \frac{\left(\begin{array}{ll}
i & r \\
k & r
\end{array}\right)_{6}}{\left(\begin{array}{l}
r \\
r
\end{array}\right)_{6}} E_{00 j}^{r}\right. \\
& +2 \frac{\left(\begin{array}{ll}
j & r \\
k & r
\end{array}\right)_{6}}{\left(\begin{array}{l}
r \\
r
\end{array}\right)_{6}^{r}} E_{00 i}^{r}
\end{aligned}
$$

and with the same argument like the one used after (4.23) we obtain the final result

$$
\begin{aligned}
I_{6}^{\mu \nu \lambda \rho}= & \sum_{i, j, k=1}^{5} q_{i}^{\mu} q_{j}^{\nu} q_{k}^{\lambda} \sum_{r=1, r \neq i, j, k}^{6} v_{r}^{\rho} E_{i j k}^{r}+g^{\mu \nu} \sum_{k=1}^{5} q_{k}^{\lambda} \sum_{r=1, r \neq k}^{6} v_{r}^{\rho} E_{00 k}^{r}+g^{\mu \lambda} \sum_{j=1}^{5} q_{j}^{\nu} \sum_{r=1, r \neq j}^{6} v_{r}^{\rho} E_{00 j}^{r} \\
& +g^{\nu \lambda} \sum_{i=1}^{5} q_{i}^{\mu} \sum_{r=1, r \neq i}^{6} v_{r}^{\rho} E_{00 i}^{r},
\end{aligned}
$$

or 


$$
I_{6}^{\mu \nu \lambda \rho}=\sum_{r=1}^{6} v_{r}^{\rho} I_{5}^{\mu \nu \lambda, r}
$$

with

$$
I_{5}^{\mu \nu \lambda, r}=\sum_{i, j, k=1 ; i, j, k \neq r}^{5} q_{i}^{\mu} q_{j}^{\nu} q_{k}^{\lambda} E_{i j k}^{r}+\sum_{k=1, k \neq r}^{5} g^{[\mu \nu} q_{k}^{\lambda]} E_{00 k}^{r}
$$

\section{NUMERICAL RESULTS AND DISCUSSION}

In order to illustrate the numerical results which can be obtained with the described approach, we will evaluate a representative collection of tensor coefficients. We rely on two implementations of the formalism, one has been established in FORTRAN, and the other one in the MATHEMATICA package HEXAGON.M.

In the following, we denote the scalar five-point function by $E_{0}$ and the scalar six-point function by $F_{0}$. The tensor decompositions of pentagons $E$ and hexagons $F$ read

$$
\begin{gathered}
E^{\mu}=\sum_{i=1}^{4} q_{i}^{\mu} E_{i} \\
E^{\mu \nu}=\sum_{i, j=1}^{4} q_{i}^{\mu} q_{j}^{\nu} E_{i j}+g^{\mu \nu} E_{00} \\
E^{\mu \nu \lambda}=\sum_{i, j, k=1}^{4} q_{i}^{\mu} q_{j}^{\nu} q_{k}^{\lambda} E_{i j k}+\sum_{i=1}^{4} g^{[\mu \nu} q_{i}^{\lambda]} E_{00 i} \\
F^{\mu}=\sum_{i=1}^{5} q_{i}^{\mu} F_{i} \\
F^{\mu \nu \lambda \rho}=\sum_{i, j, k, l=1}^{5} q_{i}^{\mu} q_{j}^{\nu} q_{k}^{\lambda} q_{l}^{\rho} F_{i j k l}+\sum_{i, j=1}^{5} q_{i}^{\mu} q_{j}^{[\nu} g^{\lambda \rho]} F_{00 i j} \\
\sum_{i, j, k=1}^{5} q_{i}^{\mu} q_{j}^{\nu} q_{k}^{\lambda} F_{i j k}+\sum_{i=1}^{5} g^{\mu \nu} q_{i}^{\lambda} F_{00 i}
\end{gathered}
$$

Please observe the difference of $E^{0}, F^{0}$ and $E_{0}, F_{0}$ in the following. The kinematics is visualized in Fig. 1. Deviating from the first sections, we have chosen here $q_{0}=0$ in order to stay close to common conventions of other numerical packages.

For the evaluation of the scalar two-, three-, and fourpoint functions, which appear after the complete reduction,
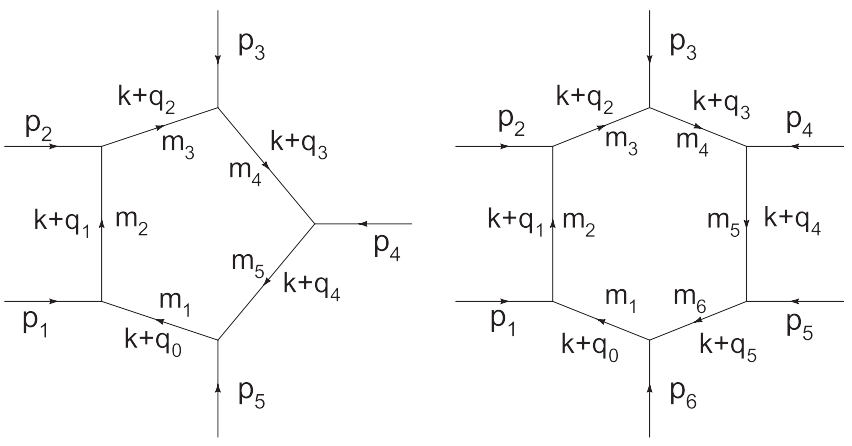

FIG. 1. Momenta flow used in the numerical examples for sixand five-point integrals.

we have implemented two numerical libraries in our FORTRAN package:

(i) For massive internal particles: LOOPTOOLS 2.2 [4,38];

(ii) If there are also massless internal particles: QCDLOOP-1.4 [40].

We observed that LOOPTOOLS may become unstable in the presence of massless internal particles. For the fully massive cases, we compared the results obtained using the two numerical libraries as a cross check. Our MATHEMATICA package has an implementation of only LOOPTOOLS.

For completeness, we would like to mention also other publicly available FORTRAN packages for tensor functions, which we found useful for comparisons:

(i) Six-point tensors with massive internal particles: none;

(ii) Five-point tensors with massive internal particles: LOOPTOOLS [4,38];

(iii) Five-point tensors with both massive and massless particles: none;

(iv) Five- and six-point tensors with only massless internal particles: GOLEM95 [43].

The two independent numerical implementations have been checked in several ways:

(i) By internal comparisons of the two codes, relying on the formulas presented in this article; With alternative, direct representations of the tensor integrals with sector decomposition [44,45] and MellinBarnes representations [46,47];

(ii) By simplifying the numerator structures algebraically and subsequent evaluation of the resulting integrals of lower rank;

(iii) By direct comparison with other tensor integral packages $[38,43]$.

Some of the comparisons were documented in [37].

We restrict ourselves to a few phase-space points, see Tables I, II, and III. The first configuration corresponds to the reaction $g g \rightarrow t \bar{t} q \bar{q}$, with external momenta generated by MADGRAPH $[48,49]$. The second configuration comes from [43], while the third is a slight modification of the first 
TABLE I. The components of external four-momenta for the six-point numerics; all internal particles are massive. For five-point functions, we shrink line 2 and fix $p_{1}+p_{2} \rightarrow p_{1}$ in order to retain momentum conservation.

\begin{tabular}{llccc}
\hline \hline$p_{1}$ & $0.21774554 \times 10^{3}$ & 0.0 & 0.0 & $0.21774554 \times 10^{3}$ \\
$p_{2}$ & $0.21774554 \times 10^{3}$ & 0.0 & 0.0 & $-0.21774554 \times 10^{3}$ \\
$p_{3}$ & $-0.20369415 \times 10^{3}$ & $-0.47579512 \times 10^{2}$ & $0.42126823 \times 10^{2}$ & $0.84097181 \times 10^{2}$ \\
$p_{4}$ & $-0.20907237 \times 10^{3}$ & $0.55215961 \times 10^{2}$ & $-0.46692034 \times 10^{2}$ & $-0.90010087 \times 10^{2}$ \\
$p_{5}$ & $-0.68463308 \times 10^{1}$ & $0.53063195 \times 10^{1}$ & $0.29698267 \times 10^{1}$ & $-0.31456871 \times 10^{1}$ \\
$p_{6}$ & $-0.15878244 \times 10^{2}$ & $-0.12942769 \times 10^{2}$ & $0.15953850 \times 10^{1}$ & $0.90585932 \times 10^{1}$ \\
& $m_{1}=110.0, m_{2}=120.0, m_{3}=130.0, m_{4}=140.0, m_{5}=150.0, m_{6}=160.0$ \\
\hline
\end{tabular}

TABLE II. The external four-momenta for the six-point numerics; all internal particles are massless. This set of momenta comes from [43]. For five-point functions, we shrink line 2 and fix $p_{1}+p_{2} \rightarrow p_{1}$ in order to retain momentum conservation.

\begin{tabular}{lcccc}
\hline \hline$p_{1}$ & 0.5 & 0.0 & 0.0 & 0.5 \\
$p_{2}$ & 0.5 & 0.0 & 0.0 & -0.5 \\
$p_{3}$ & -0.19178191 & -0.12741180 & -0.08262477 & -0.11713105 \\
$p_{4}$ & -0.33662712 & 0.06648281 & 0.31893785 & 0.08471424 \\
$p_{5}$ & -0.21604814 & 0.20363139 & -0.04415762 & -0.05710657 \\
& & $p_{6}=-\left(p_{1}+p_{2}+p_{3}+p_{4}+p_{5}\right), m_{1}=\cdots=m_{6}=0.0$ & \\
\hline \hline
\end{tabular}

TABLE III. The external four-momenta for the six-point numerics; one internal mass is finite. For five-point functions, we shrink line 2 and fix $p_{1}+p_{2} \rightarrow p_{1}$ in order to retain momentum conservation.

\begin{tabular}{lcccc}
\hline \hline$p_{1}$ & $0.21774554 \times 10^{1}$ & 0.0 & 0.0 & $0.21774554 \times 10^{1}$ \\
$p_{2}$ & $0.21774554 \times 10^{1}$ & 0.0 & 0.0 & $-0.21774554 \times 10^{1}$ \\
$p_{3}$ & $-0.20369415 \times 10^{1}$ & $-0.47579512 \times 10^{0}$ & $0.42126823 \times 10^{0}$ & $0.84097181 \times 10^{0}$ \\
$p_{4}$ & $-0.20907237 \times 10^{1}$ & $0.55215961 \times 10^{0}$ & $-0.46692034 \times 10^{0}$ & $-0.90010087 \times 10^{0}$ \\
$p_{5}$ & $-0.68463308 \times 10^{-1}$ & $0.53063195 \times 10^{-1}$ & $0.29698267 \times 10^{-1}$ & $-0.31456871 \times 10^{-1}$ \\
$p_{6}$ & $-0.15878244 \times 10^{0}$ & $-0.12942769 \times 10^{0}$ & $0.15953850 \times 10^{-1}$ & $0.90585932 \times 10^{-1}$ \\
& $m_{1}=0.0, m_{2}=0.0, m_{3}=0.0, m_{4}=1.7430, m_{5}=0.0, m_{6}=0.0$ & \\
\hline \hline
\end{tabular}

one. The kinematical input is completed by adding the masses of internal particles.

We begin with massive six-point tensors. For the kinematics introduced above, we determine the tensor components with our FORTRAN package as shown in Tables IV, V, and VI. They are complex, finite numbers. Only independent components of the tensors are shown, all the remaining ones are obtained by permutations of indices.

Selected tensor coefficients of five-point tensors for the case of massive internal particles are shown in Table VII [50]. The coefficients have been compared with LOOPTOOLS 2.2 and indeed we agree. For the massive sixpoint functions, there is no alternative package publicly available.

In presence of massless internal particles, we face potential infrared singularities. Then, the loop functions are Laurent series in $\epsilon$, starting with a term proportional to $\frac{1}{\epsilon^{2}}$, and one has to care about renormalizations compared to our basic definition (1.1). A popular measure is $[40,43]$ :

$$
\mathcal{M}=(\mu)^{4-d} \frac{\Gamma(1-2 \epsilon)}{\Gamma(1+\epsilon) \Gamma^{2}(1-\epsilon)} \int \frac{d^{d} k}{i \pi^{d / 2}} .
$$

TABLE IV. Tensor components for scalar, vector, and rank $R=2$ six-point functions; kinematics defined in Table I and Fig. 1.

\begin{tabular}{|c|c|c|}
\hline & & $\begin{array}{c}F_{0} \\
-0.223393 \times 10^{-18}-\mathrm{i} 0.396728 \times 10^{-19}\end{array}$ \\
\hline$\mu$ & & $F^{\mu}$ \\
\hline 0 & & $0.192487 \times 10^{-17}+\mathrm{i} 0.972635 \times 10^{-17}$ \\
\hline 1 & & $-0.363320 \times 10^{-17}-\mathrm{i} 0.11940 \times 10^{-17}$ \\
\hline 2 & & $0.365514 \times 10^{-17}+\mathrm{i} 0.106928 \times 10^{-17}$ \\
\hline 3 & & $0.239793 \times 10^{-16}+\mathrm{i} 0.341928 \times 10^{-17}$ \\
\hline$\mu$ & $\nu$ & $F^{\mu \nu}$ \\
\hline 0 & 0 & $0.599459 \times 10^{-14}-\mathrm{i} 0.114601 \times 10^{-14}$ \\
\hline 0 & 1 & $0.323869 \times 10^{-15}+\mathrm{i} 0.423754 \times 10^{-15}$ \\
\hline 0 & 2 & $-0.294252 \times 10^{-15}-\mathrm{i} 0.375481 \times 10^{-15}$ \\
\hline 0 & 3 & $-0.255450 \times 10^{-14}-\mathrm{i} 0.195640 \times 10^{-14}$ \\
\hline 1 & 1 & $-0.164562 \times 10^{-14}-\mathrm{i} 0.993796 \times 10^{-16}$ \\
\hline 1 & 2 & $0.920944 \times 10^{-16}+\mathrm{i} 0.706487 \times 10^{-17}$ \\
\hline 1 & 3 & $0.347694 \times 10^{-15}-\mathrm{i} 0.127190 \times 10^{-16}$ \\
\hline 2 & 2 & $-0.163339 \times 10^{-14}-\mathrm{i} 0.994148 \times 10^{-16}$ \\
\hline 2 & 3 & $-0.341773 \times 10^{-15}+\mathrm{i} 0.818678 \times 10^{-17}$ \\
\hline 3 & 3 & $-0.413909 \times 10^{-14}+\mathrm{i} 0.670676 \times 10^{-15}$ \\
\hline
\end{tabular}


TABLE V. Tensor components for a massive rank $R=3$ six-point function; kinematics defined in Table I and Figure 1.

\begin{tabular}{lllc}
\hline \hline$\mu$ & $\nu$ & $\lambda$ & $F^{\mu \nu \lambda}$ \\
\hline 0 & 0 & 0 & $-0.227754 \times 10^{-11}-\mathrm{i} 0.267244 \times 10^{-12}$ \\
0 & 0 & 1 & $0.140271 \times 10^{-13}-\mathrm{i} 0.119448 \times 10^{-12}$ \\
0 & 0 & 2 & $-0.201270 \times 10^{-13}+\mathrm{i} 0.101968 \times 10^{-12}$ \\
0 & 0 & 3 & $0.102976 \times 10^{-12}+\mathrm{i} 0.624467 \times 10^{-12}$ \\
0 & 1 & 1 & $0.183904 \times 10^{-12}+\mathrm{i} 0.142429 \times 10^{-12}$ \\
0 & 1 & 2 & $-0.131028 \times 10^{-13}-\mathrm{i} 0.610343 \times 10^{-14}$ \\
0 & 1 & 3 & $-0.543316 \times 10^{-13}-\mathrm{i} 0.158809 \times 10^{-13}$ \\
0 & 2 & 2 & $0.181352 \times 10^{-12}+\mathrm{i} 0.141686 \times 10^{-12}$ \\
0 & 2 & 3 & $0.506408 \times 10^{-13}+\mathrm{i} 0.163568 \times 10^{-13}$ \\
0 & 3 & 3 & $-0.563539 \times 10^{-13}+\mathrm{i} 0.178403 \times 10^{-13}$ \\
1 & 1 & 1 & $0.210641 \times 10^{-13}-\mathrm{i} 0.584990 \times 10^{-14}$ \\
1 & 1 & 2 & $0.120482 \times 10^{-12}-\mathrm{i} 0.574688 \times 10^{-13}$ \\
1 & 1 & 3 & $-0.201182 \times 10^{-13}+\mathrm{i} 0.620591 \times 10^{-14}$ \\
1 & 2 & 2 & $-0.686164 \times 10^{-14}+\mathrm{i} 0.205457 \times 10^{-14}$ \\
1 & 2 & 3 & $0.582201 \times 10^{-13}-\mathrm{i} 0.163889 \times 10^{-13}$ \\
1 & 3 & 3 & $0.119659 \times 10^{-12}-\mathrm{i} 0.570084 \times 10^{-13}$ \\
2 & 2 & 2 & $0.457464 \times 10^{-13}-\mathrm{i} 0.181141 \times 10^{-13}$ \\
2 & 2 & 3 & $0.557081 \times 10^{-12}-\mathrm{i} 0.374359 \times 10^{-12}$ \\
2 & 3 & 3 & \\
3 & 3 & 3 &
\end{tabular}

TABLE VI. Tensor components for a massive rank $R=4$ six-point function; kinematics defined in Table I and Fig. 1 .

\begin{tabular}{|c|c|c|c|c|c|c|c|c|c|}
\hline$\mu$ & $\nu$ & $\lambda$ & $\rho$ & $F^{\mu \nu \lambda \rho}$ & $\mu$ & $\nu$ & $\lambda$ & $\rho$ & $F^{\mu \nu \lambda \rho}$ \\
\hline 0 & 0 & 0 & 0 & $0.666615 \times 10^{-9}+\mathrm{i} 0.247562 \times 10^{-9}$ & 0 & 2 & 3 & 3 & $-0.802359 \times 10^{-11}+\mathrm{i} 0.994701 \times 10^{-12}$ \\
\hline 0 & 0 & 0 & 1 & $-0.200049 \times 10^{-10}+\mathrm{i} 0.294036 \times 10^{-10}$ & 0 & 3 & 3 & 3 & $-0.102576 \times 10^{-9}+\mathrm{i} 0.378476 \times 10^{-10}$ \\
\hline 0 & 0 & 0 & 2 & $0.200975 \times 10^{-10}-\mathrm{i} 0.237333 \times 10^{-10}$ & 1 & 1 & 1 & 1 & $-0.246426 \times 10^{-10}+\mathrm{i} 0.276326 \times 10^{-10}$ \\
\hline 0 & 0 & 0 & 3 & $0.645477 \times 10^{-10}-\mathrm{i} 0.162236 \times 10^{-9}$ & 1 & 1 & 1 & 2 & $0.915670 \times 10^{-12}-\mathrm{i} 0.660629 \times 10^{-12}$ \\
\hline 0 & 0 & 1 & 1 & $-0.116956 \times 10^{-10}-\mathrm{i} 0.516760 \times 10^{-10}$ & 1 & 1 & 1 & 3 & $0.303529 \times 10^{-11}-\mathrm{i} 0.287480 \times 10^{-11}$ \\
\hline 0 & 0 & 1 & 2 & $0.160357 \times 10^{-11}+\mathrm{i} 0.222284 \times 10^{-11}$ & 1 & 1 & 2 & 2 & $-0.822697 \times 10^{-11}+\mathrm{i} 0.919635 \times 10^{-11}$ \\
\hline 0 & 0 & 1 & 3 & $0.792692 \times 10^{-11}+\mathrm{i} 0.729502 \times 10^{-11}$ & 1 & 1 & 2 & 3 & $-0.116294 \times 10^{-11}+\mathrm{i} 0.100024 \times 10^{-11}$ \\
\hline 0 & 0 & 2 & 2 & $-0.111838 \times 10^{-10}-\mathrm{i} 0.513133 \times 10^{-10}$ & 1 & 1 & 3 & 3 & $-0.146918 \times 10^{-10}+\mathrm{i} 0.183799 \times 10^{-10}$ \\
\hline 0 & 0 & 2 & 3 & $-0.681086 \times 10^{-11}-\mathrm{i} 0.708933 \times 10^{-11}$ & 1 & 2 & 2 & 2 & $0.908296 \times 10^{-12}-\mathrm{i} 0.654735 \times 10^{-12}$ \\
\hline 0 & 0 & 3 & 3 & $-0.804454 \times 10^{-10}-\mathrm{i} 0.801909 \times 10^{-10}$ & 1 & 2 & 2 & 3 & $0.109510 \times 10^{-11}-\mathrm{i} 0.100875 \times 10^{-11}$ \\
\hline 0 & 1 & 1 & 1 & $0.100498 \times 10^{-10}-\mathrm{i} 0.151735 \times 10^{-13}$ & 1 & 2 & 3 & 3 & $0.717342 \times 10^{-12}-\mathrm{i} 0.557293 \times 10^{-12}$ \\
\hline 0 & 1 & 1 & 2 & $-0.348984 \times 10^{-11}-\mathrm{i} 0.195436 \times 10^{-12}$ & 1 & 3 & 3 & 3 & $0.450661 \times 10^{-11}-\mathrm{i} 0.485065 \times 10^{-11}$ \\
\hline 0 & 1 & 1 & 3 & $-0.211111 \times 10^{-10}+\mathrm{i} 0.295212 \times 10^{-11}$ & 2 & 2 & 2 & 2 & $-0.245154 \times 10^{-10}+\mathrm{i} 0.274313 \times 10^{-10}$ \\
\hline 0 & 1 & 2 & 2 & $0.357455 \times 10^{-11}+\mathrm{i} 0.662809 \times 10^{-14}$ & 2 & 2 & 2 & 3 & $-0.318500 \times 10^{-11}+\mathrm{i} 0.279750 \times 10^{-11}$ \\
\hline 0 & 1 & 2 & 3 & $0.121595 \times 10^{-11}-\mathrm{i} 0.807388 \times 10^{-13}$ & 2 & 2 & 3 & 3 & $-0.146317 \times 10^{-10}+\mathrm{i} 0.182912 \times 10^{-10}$ \\
\hline 0 & 1 & 3 & 3 & $0.825803 \times 10^{-11}-\mathrm{i} 0.142086 \times 10^{-11}$ & 2 & 3 & 3 & 3 & $-0.477335 \times 10^{-11}+\mathrm{i} 0.477368 \times 10^{-11}$ \\
\hline 0 & 2 & 2 & 2 & $-0.958961 \times 10^{-11}-\mathrm{i} 0.585948 \times 10^{-12}$ & 3 & 3 & 3 & 3 & $-0.730168 \times 10^{-10}+\mathrm{i} 0.112865 \times 10^{-9}$ \\
\hline 0 & 2 & 2 & 3 & $-0.209232 \times 10^{-10}+\mathrm{i} 0.289031 \times 10^{-11}$ & & & & & \\
\hline
\end{tabular}

TABLE VII. Selected tensor coefficients of five-point tensor functions with massive internal particles; kinematics defined in Table I.

\begin{tabular}{ll}
\hline \hline$E_{0}$ & $0.702503 \times 10^{-14}+\mathrm{i} 0.170006 \times 10^{-14}$ \\
$E_{1}$ & $3.56379 \times 10^{-15}-\mathrm{i} 5.58904 \times 10^{-16}$ \\
$E_{12}$ & $-7.86411 \times 10^{-16}+\mathrm{i} 1.03994 \times 10^{-15}$ \\
$E_{00}$ & $-8.18587 \times 10^{-11}+\mathrm{i} 1.80354 \times 10^{-11}$ \\
$E_{123}$ & $3.51267 \times 10^{-16}+\mathrm{i} 9.64413 \times 10^{-17}$ \\
$E_{001}$ & $9.38702 \times 10^{-12}+\mathrm{i} 2.18811 \times 10^{-11}$ \\
\hline
\end{tabular}


TABLE VIII. Tensor coefficients $F_{0}, F_{3}, F_{22}$ and tensor components $F^{000}, F^{1111}$ of six-point functions; all internal particles are massless, kinematics of Table II.

\begin{tabular}{lccc}
\hline \hline & $\epsilon^{0}$ & $1 / \epsilon$ & $1 / \epsilon^{2}$ \\
\hline$F_{0}$ & $-57.8724994-\mathrm{i} 9248.84583$ & $-3167.69411-\mathrm{i} 2981.57728$ & -1003.89197 \\
$F_{3}$ & $-867.761166+\mathrm{i} 859.212722$ & $273.495904+\mathrm{i} 483.076108$ & 153.767901 \\
$F_{22}$ & $83.1234074-\mathrm{i} 271.20343$ & $-75.7263181-\mathrm{i} 95.1508846$ & -30.2874673 \\
$F^{000}$ & $-185.635891+\mathrm{i} 1465.754753$ & $487.259427+\mathrm{i} 525.6914058$ & 174.2745041 \\
$F^{111}$ & $-2.64116950-\mathrm{i} 4.28827971$ & $-0.8480346995-\mathrm{i} 0.4557274228$ & -0.1450625441 \\
\hline \hline
\end{tabular}

TABLE IX. Selected tensor coefficients of five-point tensor functions with massless internal particles; kinematics defined in Table II.

\begin{tabular}{lccc}
\hline \hline & $\epsilon^{0}$ & $1 / \epsilon$ & $1 / \epsilon^{2}$ \\
\hline$E_{0}$ & $202.168496+\mathrm{i} 3211.04072$ & $1022.10601+\mathrm{i} 972.027061$ & $309.405823+\mathrm{i} 0.0$ \\
$E_{2}$ & $264.996441-\mathrm{i} 303.068452$ & $-96.4696846-\mathrm{i} 149.228472$ & $-47.5008979+\mathrm{i} 0.0$ \\
$E_{33}$ & $1780.58042+\mathrm{i} 2914.50734$ & $927.71650+\mathrm{i} 568.572069$ & $180.982111+\mathrm{i} 0.0$ \\
$E_{00}$ & $9.56327810+\mathrm{i} 1.61648472 \times 10^{-13}$ & $4.70734562 \times 10^{-14}+\mathrm{i} 2.48689958 \times 10^{-14}$ & $7.10542736 \times 10^{-15}+\mathrm{i} 0.0$ \\
$E_{444}$ & $-1035.29689-\mathrm{i} 1422.01085$ & $-452.640112-\mathrm{i} 254.226520$ & $-80.9228146+\mathrm{i} 0.0$ \\
$E_{001}$ & $-0.81227772-\mathrm{i} 5.68434189 \times 10^{-14}$ & $-2.04281037 \times 10^{-14}-\mathrm{i} 2.84217094 \times 10^{-14}$ & $-7.10542736 \times 10^{-15}+\mathrm{i} 0.0$ \\
\hline \hline
\end{tabular}

When discussing Feynman integrals with a dependence on inverse powers of $\epsilon$ there appears a dependence of their constant terms on these conventions. For convenience of the reader, the tables are produced with a normalization as introduced in Eq. (5.8), with the choice $\mu=1$.

For the case of six-point and five-point functions with only massless internal particles, we show only a few sample coefficients in Tables IX and VIII, which are produced with our FORTRAN package. The phase-space point chosen here is defined in Table II. We checked that, within double precision, we completely agree with corresponding numbers produced with GOLEM95.
Finally, to complete the list of relevant results, we show also sample tensor coefficients for the case of both massive and massless internal particles, for five-point tensors in Table X and for six-point tensors in Table XI. For this case with mixed internal masses, there is no other publicly released code available.

To summarize, in this article, we have presented tensor integrals of rank $R \leq 3$ for five-point functions and of rank $R \leq 4$ for six-point functions. These ranks are sufficient for the calculation of e.g. one-loop QCD corrections to four fermion production at the LHC. Infrared divergent kinematical configurations are treated using dimensional regu-

TABLE X. Selected tensor components of five-point tensor functions with both massive and massless internal particles; kinematics defined in Table III.

\begin{tabular}{lccc}
\hline \hline & $\epsilon^{0}$ & $1 / \epsilon$ & $1 / \epsilon^{2}$ \\
\hline$E_{0}$ & $-0.289852933 \times 10^{4}+\mathrm{i} 0.228935552 \times 10^{3}$ & $-0.945038648 \times 10^{2}+\mathrm{i} 0.454178453 \times 10^{2}$ & $0.7112330546 \times 10^{1}+\mathrm{i} 0.0$ \\
$E^{3}$ & $0.168344624 \times 10^{3}-\mathrm{i} 0.181758172 \times 10^{2}$ & $0.4242553725 \times 10^{1}-\mathrm{i} 0.338838829 \times 10^{1}$ & $-0.6442770877 \times 10^{0}+\mathrm{i} 0.0$ \\
$E^{23}$ & $-0.79409571852 \times 10^{1}+\mathrm{i} 0.5445326927 \times 10^{0}$ & $-0.3008645503 \times 10^{0}+\mathrm{i} 0.9457613783 \times 10^{-1}$ & $0.1027869989 \times 10^{-1}+\mathrm{i} 0.0$ \\
$E^{012}$ & $0.2472148936 \times 10^{1}-\mathrm{i} 0.127011969 \times 10^{0}$ & $0.9699262574 \times 10^{-1}-\mathrm{i} 0.2560545796 \times 10^{-1}$ & $-0.2331885086 \times 10^{-2}+\mathrm{i} 0.0$ \\
$E^{2130}$ & $0.2733228280 \times 10^{2}-\mathrm{i} 0.519106421 \times 10^{2}$ & $-0.909476582 \times 10^{1}+\mathrm{i} 0.1744459753 \times 10^{-2}$ & $0.2112313083 \times 10^{-3}+\mathrm{i} 0.0$ \\
\hline \hline
\end{tabular}

TABLE XI. Selected tensor components of six-point tensor functions with both massive and massless internal particles; kinematics defined in Table III.

\begin{tabular}{lcccc}
\hline \hline & $\epsilon^{0}$ & $1 / \epsilon$ & $1 / \epsilon^{2}$ \\
\hline$F_{0}$ & $0.2403558675 \times 10^{4}-\mathrm{i} 0.2058213187 \times 10^{3}$ & $0.7315208677 \times 10^{2}-\mathrm{i} 0.4276718518 \times 10^{2}$ & $-0.7543148872 \times 10^{1}+\mathrm{i} 0.0$ \\
$F^{2}$ & $0.1112747404 \times 10^{3}-\mathrm{i} 0.6809282900 \times 10^{1}$ & $0.4419243474 \times 10^{1}-\mathrm{i} 0.1201033663 \times 10^{1}$ & $-0.1044856909 \times 10^{0}+\mathrm{i} 0.0$ \\
$F^{13}$ & $-0.1014018623 \times 10^{2}+\mathrm{i} 0.1797332619 \times 10^{1}$ & $-0.5914958485 \times 10^{-1}+\mathrm{i} 0.3275539398 \times 10^{0}$ & $0.7678550480 \times 10^{-1}+\mathrm{i} 0.0$ \\
$F^{123}$ & $-0.5007216712 \times 10^{0}+\mathrm{i} 0.4194342396 \times 10^{-1}$ & $-0.1642316924 \times 10^{-1}+\mathrm{i} 0.7789453935 \times 10^{-2}$ & $0.1225024390 \times 10^{-2}+\mathrm{i} 0.0$ \\
$F^{3210}$ & $0.1263455978 \times 10^{0}-\mathrm{i} 0.6509987460 \times 10^{-2}$ & $0.4610567958 \times 10^{-2}-\mathrm{i} 0.1506637282 \times 10^{-2}$ & $-0.1945123881 \times 10^{-3}+\mathrm{i} 0.0$ \\
\hline \hline
\end{tabular}


larization. The final reduction formulas are valid up to terms of order $\epsilon$, which do not contribute to physical quantities in applications at NLO.

There are further reactions of interest which will need higher-point functions and higher ranks of five- and sixpoint functions. The details of their reductions have been left for a later investigation.

\section{ACKNOWLEDGMENTS}

Work supported by Sonderforschungsbereich/ Transregio SFB/TRR 9 of DFG "Computergestützte Theoretische Teilchenphysik" and by the European Community's Marie-Curie Research Training Networks MRTN-CT-2006-035505 HEPTOOLS and MRTN-CT2006-035482 FLAVIAnet. J. G. was supported by MNiSW, Grant No. N202 104936. K. K. acknowledges the UPGOW project cofinanced by the European Social Fund. J. F. likes to thank DESY for the kind hospitality. We thank Th. Binoth, A. Denner, S. Dittmaier, Th. Hahn, C. Papadopoulos and P. Uwer for useful discussions.

\section{APPENDIX A: GRAM DETERMINANTS AND ALGEBRA OF SIGNED MINORS}

In this section relations are derived, which will turn out to be indispensable in our tensor reductions.

We begin with some notational remarks on Gram determinants $G_{n-1}$,

$$
G_{n-1}=\left|2 q_{j} q_{k}\right|, \quad j, k=1, \cdots, n-1 .
$$

The modified Cayley determinant of a diagram with $n$ internal lines with chords $q_{j}$ is

$$
\begin{aligned}
()_{n} & =\left|C_{j k}\right|, j, k=0, \cdots, n, \\
& =\left|\begin{array}{ccccc}
0 & 1 & 1 & \ldots & 1 \\
1 & Y_{11} & Y_{12} & \ldots & Y_{1 n} \\
1 & Y_{12} & Y_{22} & \ldots & Y_{2 n} \\
\vdots & \vdots & \vdots & \ddots & \vdots \\
1 & Y_{1 n} & Y_{2 n} & \ldots & Y_{n n}
\end{array}\right|,
\end{aligned}
$$

with

$$
Y_{j k}=-\left(q_{j}-q_{k}\right)^{2}+m_{j}^{2}+m_{k}^{2} .
$$

From our choice $q_{n}=0$, it follows that both determinants are related:

$$
()_{n}=-G_{n-1},
$$

and we will usually call ()$_{n}$ the Gram determinant of the Feynman integral.

Signed minors [25] are determinants (with a sign convention) which are obtained by excluding rows and columns from the modified Cayley determinant ()$_{n}$. They are denoted by the symbol

$$
\left(\begin{array}{llll}
j_{1} & j_{2} & \cdots & j_{m} \\
k_{1} & k_{2} & \cdots & k_{m}
\end{array}\right),
$$

labeling the rows $j_{1}, j_{2}, \cdots, j_{m}$ and columns $k_{1}, k_{2}, \cdots, k_{m}$ which have been excluded from ()$_{n}$. The sign of a signed minor is defined by

$$
\begin{aligned}
& (-1)^{j_{1}+j_{2}+\cdots+j_{m}+k_{1}+k_{2}+\cdots+k_{m}} \times \text { Signature }\left[j_{1}, j_{2}, \cdots j_{m}\right] \\
& \quad \times \text { Signature }\left[k_{1}, k_{2}, \cdots k_{m}\right],
\end{aligned}
$$

where Signature gives the sign of permutations to place the indices in increasing order. This agrees e.g. with the definition of the operator Signature[List] in MATHEMATICA. As an example may serve the quantity $\Delta_{n}$ :

$$
\Delta_{n}=\left|\begin{array}{cccc}
Y_{11} & Y_{12} & \ldots & Y_{1 n} \\
Y_{12} & Y_{22} & \ldots & Y_{2 n} \\
\vdots & \vdots & \ddots & \vdots \\
Y_{1 n} & Y_{2 n} & \ldots & Y_{n n}
\end{array}\right|=\left(\begin{array}{l}
0 \\
0
\end{array}\right)_{n}
$$

We now will derive two relations between signed minors. Let us introduce

$$
\begin{aligned}
A_{i j}^{s} \equiv & -\left(\begin{array}{l}
0 \\
j
\end{array}\right)_{5}\left(\begin{array}{ll}
0 & s \\
0 & i
\end{array}\right)_{5}\left(\begin{array}{l}
s \\
s
\end{array}\right)_{5}-\left(\begin{array}{l}
s \\
j
\end{array}\right)_{5}\left(\begin{array}{ll}
0 & s \\
i & s
\end{array}\right)_{5}\left(\begin{array}{l}
0 \\
0
\end{array}\right)_{5} \\
& +\left(\begin{array}{l}
0 \\
s
\end{array}\right)_{5}\left(\begin{array}{ll}
0 & s \\
0 & s
\end{array}\right)_{5}\left(\begin{array}{l}
i \\
j
\end{array}\right)_{5} .
\end{aligned}
$$

We are going to show that this expression can be factorized as

$$
A_{i j}^{s}=()_{5} X_{i j}^{s},
$$

and provide an explicit expression for $X_{i j}^{s}$. To begin with, we show that $A_{i j}^{s}$ is symmetric in the indices $i$ and $j$ for fixed $s$. Obviously the third term on the right-hand side of (A8) is symmetric since we consider a symmetric determinant. The symmetry of the first two terms means

$$
\begin{aligned}
& \left(\begin{array}{l}
s \\
s
\end{array}\right)_{5}\left[\left(\begin{array}{l}
0 \\
i
\end{array}\right)_{5}\left(\begin{array}{ll}
0 & j \\
0 & s
\end{array}\right)_{5}-\left(\begin{array}{l}
0 \\
j
\end{array}\right)_{5}\left(\begin{array}{ll}
0 & i \\
0 & s
\end{array}\right)_{5}\right] \\
& +\left(\begin{array}{l}
0 \\
0
\end{array}\right)_{5}\left[\left(\begin{array}{l}
s \\
i
\end{array}\right)_{5}\left(\begin{array}{ll}
0 & s \\
j & s
\end{array}\right)_{5}-\left(\begin{array}{l}
s \\
j
\end{array}\right)_{5}\left(\begin{array}{ll}
0 & s \\
i & s
\end{array}\right)_{5}\right]=0 .
\end{aligned}
$$

The first square bracket of (A10) can be evaluated using (A.13) of [25], i.e.

$$
\left(\begin{array}{l}
0 \\
j
\end{array}\right)_{5}\left(\begin{array}{ll}
0 & i \\
0 & s
\end{array}\right)_{5}=-\left(\begin{array}{l}
0 \\
0
\end{array}\right)_{5}\left(\begin{array}{ll}
0 & s \\
i & j
\end{array}\right)_{5}+\left(\begin{array}{l}
0 \\
i
\end{array}\right)_{5}\left(\begin{array}{ll}
0 & j \\
0 & s
\end{array}\right)_{5}
$$

and (A10) then results in 
$\left(\begin{array}{l}s \\ i\end{array}\right)_{5}\left(\begin{array}{ll}0 & s \\ j & s\end{array}\right)_{5}+\left(\begin{array}{l}s \\ j\end{array}\right)_{5}\left(\begin{array}{ll}0 & s \\ s & i\end{array}\right)_{5}+\left(\begin{array}{l}s \\ s\end{array}\right)_{5}\left(\begin{array}{ll}0 & s \\ i & j\end{array}\right)_{5}=0$.

This is proved by multiplication [51] with ()$_{5}$ and using Eq. (A.8) of [25] with $r=2$, i.e.

$$
\begin{gathered}
\left(\begin{array}{ll}
i & l \\
j & k
\end{array}\right)_{5}()_{5}=\left(\begin{array}{l}
i \\
j
\end{array}\right)_{5}\left(\begin{array}{l}
l \\
k
\end{array}\right)_{5}-\left(\begin{array}{l}
i \\
k
\end{array}\right)_{5}\left(\begin{array}{l}
l \\
j
\end{array}\right)_{5} \\
i, j, k, l=0, \ldots, 5 .
\end{gathered}
$$

Inserting this, products of three factors of the form

$$
\left(\begin{array}{l}
i \\
k
\end{array}\right)_{5}
$$

cancel by pairs, q.e.d.

For the following, relations (A.11) and (A.12) of [25] become important, i.e.

$$
\sum_{i=1}^{n}\left(\begin{array}{l}
0 \\
i
\end{array}\right)_{5}=()_{5}
$$

and

$$
\sum_{i=1}^{n}\left(\begin{array}{l}
j \\
i
\end{array}\right)_{5}=0, \quad(j \neq 0) .
$$

Further, "extensionals" are needed, i.e. relations valid for ()$_{5}$ can be extended to any minor of ()$_{5}$; an extensional of (A14) e.g. is

$$
\sum_{i=1}^{n}\left(\begin{array}{ll}
j & 0 \\
k & i
\end{array}\right)_{5}=\left(\begin{array}{l}
j \\
k
\end{array}\right)_{5}
$$

As the simplest case we now immediately obtain from (A8) $A_{s s}^{s}=0$, i.e.

$$
X_{s s}^{s}=0 .
$$

Applying (A14) and (A15) to (A8), we see

$$
\sum_{j=1}^{5} A_{i j}^{s}=-()_{5}\left(\begin{array}{ll}
0 & s \\
0 & i
\end{array}\right)_{5}\left(\begin{array}{l}
s \\
s
\end{array}\right)_{5}
$$

and due to the symmetry in $i$ and $j$ we also have

$$
\sum_{i=1}^{5} A_{i j}^{s}=-()_{5}\left(\begin{array}{ll}
0 & s \\
0 & j
\end{array}\right)_{5}\left(\begin{array}{l}
s \\
s
\end{array}\right)_{5}
$$

which gives us a hint of how $X_{i j}^{s}$ might look, namely, due to (A18) it should contain a term

$$
-\left(\begin{array}{ll}
0 & s \\
0 & i
\end{array}\right)_{5}\left(\begin{array}{ll}
0 & s \\
j & s
\end{array}\right)_{5} .
$$

A further contribution must vanish after summing over $i$. Because of (A17) it must contain a factor

$$
\left(\begin{array}{ll}
0 & j \\
s & i
\end{array}\right)_{5}
$$

[52]. The second factor of this contribution can only depend on $s$ and has been determined by explicit calculation to be

$$
\left(\begin{array}{ll}
0 & s \\
0 & s
\end{array}\right)_{5}
$$

Thus we conclude

$$
X_{j i}^{s}=X_{i j}^{s}=-\left(\begin{array}{cc}
0 & s \\
0 & i
\end{array}\right)_{5}\left(\begin{array}{ll}
0 & s \\
j & s
\end{array}\right)_{5}+\left(\begin{array}{ll}
0 & j \\
s & i
\end{array}\right)_{5}\left(\begin{array}{ll}
0 & s \\
0 & s
\end{array}\right)_{5} .
$$

We come now to the second relation between signed minors. While (A20) will be needed for the reduction of 4point tensors to scalars $I_{4}^{s}$, for the reduction of 3-point tensors to scalars $I_{3}^{s t}$ we also need

$$
\begin{gathered}
-\left(\begin{array}{l}
0 \\
j
\end{array}\right)_{5}\left(\begin{array}{ll}
t & s \\
0 & i
\end{array}\right)_{5}\left(\begin{array}{l}
s \\
s
\end{array}\right)_{5}-\left(\begin{array}{l}
s \\
j
\end{array}\right)_{5}\left(\begin{array}{ll}
t & s \\
i & s
\end{array}\right)_{5}\left(\begin{array}{l}
0 \\
0
\end{array}\right)_{5} \\
+\left(\begin{array}{l}
0 \\
s
\end{array}\right)_{5}\left(\begin{array}{ll}
t & s \\
0 & s
\end{array}\right)_{5}\left(\begin{array}{l}
i \\
j
\end{array}\right)_{5}=()_{5} X_{i j}^{s t},
\end{gathered}
$$

where again we have to show that indeed ()$_{5}$ factorizes and we have to give an explicit expression for $X_{i j}^{s t}$. The leftmost term on the left-hand side is an auxiliary term. It is antisymmetric in $s$ and $t$ after the cancellation of

$$
\left(\begin{array}{l}
s \\
s
\end{array}\right)_{5}
$$

and vanishes after summation over $s$ and $t$ because $I_{3}^{s t}$ is symmetric in $s$ and $t$. The cancellation of

$$
\left(\begin{array}{l}
s \\
s
\end{array}\right)_{5}
$$

has to be checked explicitly in every case where (A21) is applied.

We observe that the expressions for $X_{i j}^{s}$ (A8) and $X_{i j}^{s t}$ (A21) differ only by replacing one 0 by $t$. Therefore, the following ansatz is implied for $X_{i j}^{s t}$.

$$
X_{i j}^{s t}=-\left(\begin{array}{cc}
0 & s \\
0 & j
\end{array}\right)_{5}\left(\begin{array}{cc}
t & s \\
i & s
\end{array}\right)_{5}+\left(\begin{array}{cc}
0 & i \\
s & j
\end{array}\right)_{5}\left(\begin{array}{cc}
t & s \\
0 & s
\end{array}\right)_{5} .
$$

Now we directly evaluate $X_{i j}^{s t}()_{5}$ using (A13):

$$
\begin{aligned}
X_{i j}^{s t}()_{5}= & -\left[\left(\begin{array}{l}
0 \\
0
\end{array}\right)_{5}\left(\begin{array}{l}
s \\
j
\end{array}\right)_{5}-\left(\begin{array}{l}
s \\
0
\end{array}\right)_{5}\left(\begin{array}{l}
0 \\
j
\end{array}\right)_{5}\right]\left(\begin{array}{ll}
t & s \\
i & s
\end{array}\right) \\
& +\left[\left(\begin{array}{l}
0 \\
s
\end{array}\right)_{5}\left(\begin{array}{l}
i \\
j
\end{array}\right)_{5}-\left(\begin{array}{l}
i \\
s
\end{array}\right)_{5}\left(\begin{array}{l}
0 \\
j
\end{array}\right)_{5}\right]\left(\begin{array}{ll}
t & s \\
0 & s
\end{array}\right)
\end{aligned}
$$

and the remaining equation to be verified is 


$$
\begin{aligned}
& \left(\begin{array}{l}
s \\
0
\end{array}\right)_{5}\left(\begin{array}{l}
0 \\
j
\end{array}\right)_{5}\left(\begin{array}{ll}
t & s \\
i & s
\end{array}\right)-\left(\begin{array}{l}
i \\
s
\end{array}\right)_{5}\left(\begin{array}{l}
0 \\
j
\end{array}\right)_{5}\left(\begin{array}{ll}
t & s \\
0 & s
\end{array}\right) \\
& =-\left(\begin{array}{l}
0 \\
j
\end{array}\right)_{5}\left(\begin{array}{ll}
t & s \\
0 & i
\end{array}\right)\left(\begin{array}{l}
s \\
s
\end{array}\right)_{5},
\end{aligned}
$$

which is done by multiplying again with ()$_{5}$ and again using (A13). This gives us at the same time also a more general proof for $X_{i j}^{s}$ (A20), putting $t=0$.

\section{APPENDIX B: REDUCTION OF DIMENSIONALLY SHIFTED FIVE- AND FOUR-POINT INTEGRALS}

In this appendix we provide explicitly the needed recursion relations for the reduction of the five- and four-point functions. In spite of the fact that here, essentially, only two different relations of [29] are applied for different indices and dimension, namely, (I.30) and (I.31), we consider it helpful and sometimes even necessary, to provide them in detail. A special case of (I.31) is (B4). The others are special cases of (I.30). For the six-point function relation (I.26) plays a major role and will be quoted when applied.

$$
\begin{aligned}
\nu_{i j k} I_{5, i j k}^{[d+]^{3}}= & -\frac{\left(\begin{array}{l}
0 \\
k
\end{array}\right)_{5}}{()_{5}} I_{5, i j}^{[d++]^{2}}+\sum_{s=1, s \neq i, j}^{5} \frac{\left(\begin{array}{l}
s \\
k
\end{array}\right)_{5}}{()_{5}} I_{4, i j}^{[d+]^{2}, s} \\
& +\frac{\left(\begin{array}{l}
i \\
k
\end{array}\right)_{5}}{()_{5}} I_{5, j}^{[d+]^{2}}+\frac{\left(\begin{array}{l}
j \\
k
\end{array}\right)_{5}}{()_{5}} I_{5, i}^{[d+]^{2}},
\end{aligned}
$$

$$
\begin{aligned}
\nu_{i j} I_{5, i j}^{[d+]^{2}}= & -\frac{\left(\begin{array}{l}
0 \\
j
\end{array}\right)_{5}}{()_{5}} I_{5, i}^{[d+]}+\sum_{s=1, s \neq i}^{5} \frac{\left(\begin{array}{l}
s \\
j
\end{array}\right)_{5}}{()_{5}} I_{4, i}^{[d+], s} \\
& +\frac{\left(\begin{array}{l}
i \\
j
\end{array}\right)_{5}}{()_{5}} I_{5}^{[d+]} .
\end{aligned}
$$

The four-point function's shift is (I.44):

$$
I_{4, i}^{[d+], s}=-\frac{\left(\begin{array}{cc}
0 & s \\
i & s
\end{array}\right)_{5}}{\left(\begin{array}{l}
s \\
s
\end{array}\right)_{5}} I_{4}^{s}+\sum_{t=1, t \neq s}^{5} \frac{\left(\begin{array}{ll}
t & s \\
i & s
\end{array}\right)_{5}}{\left(\begin{array}{l}
s \\
s
\end{array}\right)_{5}} I_{3}^{s t},
$$

and the four-point integrals occurring in the reduction are (I.50):

$$
I_{4}^{[d+], s}=\left[\frac{\left(\begin{array}{cc}
0 & s \\
0 & s
\end{array}\right)_{5}}{\left(\begin{array}{l}
s \\
s
\end{array}\right)_{5}} I_{4}^{s}-\sum_{t=1, t \neq s}^{5} \frac{\left(\begin{array}{ll}
t & s \\
0 & s
\end{array}\right)_{5}}{\left(\begin{array}{l}
s \\
s
\end{array}\right)_{5}} I_{3}^{s t}\right] \frac{1}{d-3}
$$

In applications we can put $d=4$ since $I_{4}^{[d+]}$ is UV- and IRfinite. Beyond that, as it is done frequently [8], $I_{4}^{[d+]}$ can be used as well as a "master integral" [see e.g. (3.14)] without reduction to the generic dimension.
[1] G. 't Hooft and M. Veltman, Nucl. Phys. B153, 365 (1979).

[2] G. Passarino and M. Veltman, Nucl. Phys. B160, 151 (1979).

[3] G. van Oldenborgh, Comput. Phys. Commun. 66, 1 (1991).

[4] T. Hahn and M. Perez-Victoria, Comput. Phys. Commun. 118, 153 (1999).

[5] A. Denner and S. Dittmaier, Nucl. Phys. B658, 175 (2003).

[6] A. Denner and S. Dittmaier, Nucl. Phys. B734, 62 (2006).

[7] T. Binoth, J. Guillet, and G. Heinrich, Nucl. Phys. B572, 361 (2000).

[8] T. Binoth, J. Guillet, G. Heinrich, E. Pilon, and C. Schubert, J. High Energy Phys. 10 (2005) 015.

[9] A. Ferroglia, M. Passera, G. Passarino, and S. Uccirati, Nucl. Phys. B650, 162 (2003).

[10] Y. Kurihara and T. Kaneko, Comput. Phys. Commun. 174, 530 (2006).

[11] G. Ossola, C. Papadopoulos, and R. Pittau, Nucl. Phys. B763, 147 (2007).

[12] G. Ossola, C. Papadopoulos, and R. Pittau, J. High Energy Phys. 03 (2008) 042.
[13] R. K. Ellis, W. Giele, and G. Zanderighi, Phys. Rev. D 73, 014027 (2006).

[14] Z. Bern, L. Dixon, and D. Kosower, Annu. Rev. Nucl. Part. Sci. 46, 109 (1996).

[15] L. Dixon, arXiv:hep-ph/9601359.

[16] Z. Bern, L. Dixon, and D. Kosower, Ann. Phys. (N.Y.) 322, 1587 (2007).

[17] W. T. Giele and G. Zanderighi, J. High Energy Phys. 06 (2008) 038

[18] R. K. Ellis, K. Melnikov, and G. Zanderighi, J. High Energy Phys. 04 (2009) 077.

[19] C.F. Berger et al., Phys. Rev. Lett. 102, 222001 (2009).

[20] S. Weinzierl, Proc. Sci., ACAT (2007) 005.

[21] Z. Bern et al. (NLO Multileg Working Group), arXiv:0803.0494.

[22] J. Blümlein, S. Moch, and T. Riemann, Nucl. Phys. B, Proc. Suppl. 183 (2008).

[23] T. Binoth, arXiv:0903.1876.

[24] A. van Hameren, C. G. Papadopoulos, and R. Pittau, arXiv:0903.4665.

[25] D. B. Melrose, Nuovo Cimento 40, 181 (1965).

[26] A. Davydychev, Phys. Lett. B 263, 107 (1991). 
[27] K. Chetyrkin and F. Tkachov, Nucl. Phys. B192, 159 (1981).

[28] O. Tarasov, Phys. Rev. D 54, 6479 (1996).

[29] J. Fleischer, F. Jegerlehner, and O. Tarasov, Nucl. Phys. B566, 423 (2000).

[30] We will extensively quote from article [29], so we introduce here the notation (I.num) for a reference to equation (num) there.

[31] G. Duplancic and B. Nizic, Eur. Phys. J. C 35, 105 (2004).

[32] F. Jegerlehner and O. Tarasov, Nucl. Phys. B, Proc. Suppl. 116, 83 (2003).

[33] J. Campbell, E. W. N. Glover, and D. Miller, Nucl. Phys. B498, 397 (1997).

[34] G. Devaraj and R.G. Stuart, Nucl. Phys. B519, 483 (1998).

[35] J. Fleischer, J. Gluza, K. Kajda, and T. Riemann, Acta Phys. Pol. B 38, 3529 (2007).

[36] Silesian Univ., Katowice, webpage http://www.us.edu.pl/ $\sim$ gluza/hexagon, DESY, webpage http://www-zeuthen. desy.de/theory/research/CAS.html..

[37] T. Diakonidis, J. Fleischer, J. Gluza, K. Kajda, T. Riemann, and J. B. Tausk, Nucl. Phys. B, Proc. Suppl. 183, 109 (2008).

[38] T. Hahn and M. Rauch, Nucl. Phys. B, Proc. Suppl. 157, 236 (2006).

[39] A. van Hameren, J. Vollinga, and S. Weinzierl, Eur. Phys. J. C 41, 361 (2005).

[40] R. K. Ellis and G. Zanderighi, J. High Energy Phys. 02 (2008) 002.

[41] Z. Bern, L. Dixon, and D. Kosower, Nucl. Phys. B412, 751 (1994).

[42] The $I_{5, i}$ should not be confused with quantities introduced in Eq. (2.5).

[43] T. Binoth, J. P. Guillet, G. Heinrich, E. Pilon, and T. Reiter, arXiv:0810.0992.
[44] C. Bogner and S. Weinzierl, Comput. Phys. Commun. 178, 596 (2008).

[45] We used a MATHEMATICA interface to the GINAC package SECTOR_DECOMPOSITION in order to have a convenient way to evaluate tensor Feynman integrals.

[46] J. Gluza, K. Kajda, and T. Riemann, Comput. Phys. Commun. 177, 879 (2007).

[47] M. Czakon, Comput. Phys. Commun. 175, 559 (2006).

[48] T. Stelzer and W. Long, Comput. Phys. Commun. 81, 357 (1994).

[49] F. Maltoni and T. Stelzer, J. High Energy Phys. 02 (2003) 027.

[50] Please notice that we show here five-point tensor coefficients, while in the case of six-point tensors we have shown tensor components. The tensor components are representation independent and should be preferred as numerical output. For the five-point tensors with massive internal particles, however, we have arranged for a one-toone correspondence with output of LOOPTOOLS 2.2 so it might be interesting to have, in this case, the tensor coefficients instead.

[51] Assuming here ()$_{5} \neq 0$ means no limitation since we are just looking for an algebraic relation.

[52] Observe that

$$
\sum_{j=1}^{5}\left(\begin{array}{ll}
0 & j \\
s & i
\end{array}\right)_{5}=0
$$

but

$$
\sum_{i=1}^{5}\left(\begin{array}{ll}
0 & j \\
s & i
\end{array}\right)_{5}=-\sum_{i=1}^{5}\left(\begin{array}{ll}
0 & j \\
i & s
\end{array}\right)_{5}=-\left(\begin{array}{l}
j \\
s
\end{array}\right)_{5} .
$$

